\title{
A finite-volume HLLC-based scheme for compressible interfacial flows with surface tension
}

\author{
Daniel P. Garrick ${ }^{\mathrm{a}}$, Mark Owkes ${ }^{\mathrm{b}}$, Jonathan D. Regele $\mathrm{e}^{\mathrm{a}, *}$ \\ ${ }^{a}$ Department of Aerospace Engineering \\ Iowa State University \\ Ames, IA \\ ${ }^{b}$ Department of Industrial and Mechanical Engineering \\ Montana State University \\ Bozeman, $M T$
}

\begin{abstract}
Shock waves are often used in experiments to create a shear flow across liquid droplets to study secondary atomization. Similar behavior occurs inside of supersonic combustors (scramjets) under startup conditions, but it is challenging to study these conditions experimentally. In order to investigate this phenomenon further, a numerical approach is developed to simulate compressible multiphase flows under the effects of surface tension forces. The flow field is solved via the compressible multicomponent Euler equations (i.e., the five equation model) discretized with the finite volume method on a uniform Cartesian grid. The solver utilizes a total variation diminishing (TVD) third-order Runge-Kutta method for time-marching and second order TVD spatial reconstruction. Surface tension is incorporated using the Continuum Surface Force (CSF) model. Fluxes are upwinded with a modified Harten-Lax-van Leer Contact (HLLC) approximate Riemann solver. An interface compression scheme is employed to counter numerical diffusion of the interface. The present work includes modifications to both the HLLC solver and the interface compression scheme to account for capillary force terms and the associated pressure jump across the gas-liquid interface. A simple method for numerically computing the interface curvature is developed and an acoustic scaling of the surface tension coefficient is proposed for the non-dimensionalization of the model. The model captures the surface tension induced pressure jump exactly if the exact curvature is known and is further verified with an oscillating elliptical droplet and Mach 1.47 and 3 shock-droplet interaction problems. The general characteristics of secondary atomization at a range of Weber numbers are also captured in a series of simulations.
\end{abstract}

Keywords: Compressible multiphase, HLLC, surface tension, capillary forces, conservative level set, interface capturing, interface compression

* Corresponding author 


\section{Introduction}

The current research is motivated by the importance of fuel atomization in highspeed propulsion systems. Efficient combustion is greatly dictated by the fuel-injection geometry but there is a limited understanding of the effect of design parameters on the atomization process [1] , consisting of primary and secondary atomization. Primary atomization is represented by the initial breakup of a liquid sheet or jet into smaller drops or filaments. Droplets formed during primary atomization can further deform and breakup during secondary atomization. Numerical modeling can supplement experimental insights in understanding the physical processes involved during atomization, especially in flow regimes or at length scales which are otherwise difficult or impossible to measure experimentally. As of yet, little numerical work has been performed on the effects of surface tension on the atomization process in a supersonic compressible crossflow.

The secondary atomization process has been studied in some detail both experimentally and numerically (see 2 for a thorough review) and is characterized primarily by the Weber number which relates the surface tension and inertial forces. Spurred largely by the development of the Continuum Surface Force (CSF) method [3] which incorporates surface tension into the fluid governing equations as a volume force, surface tension has been studied numerically in a wide variety of incompressible applications, e.g. [4 6]. However, numerical simulations of atomization in the high Mach number regime thus far have focused on the early stages of droplet breakup where surface tension effects are not expected to play a significant role and are neglected 7 , 8.

The multiphase nature of the flow requires an adequate description of the interface between the gas and liquid. As most applications of primary atomization occur at low Mach numbers 9], numerical modeling has largely focused on the incompressible regime where interface tracking methods [10, 11] have become popular. Many different interface tracking methods exist and generally involve some type of deforming mesh or particle tracking for the purposes of reconstructing the interface. Such methods guarantee a sharp interface which allows for natural modeling of immiscible fluids and interfacial physics. Many of these methods have been extended to compressible formulations [12 15] while recent examples with surface tension effects include the all-speed model of Chang et al. [16] and the coupled incompressible-compressible large eddy solver of Xiao et al. [17. However, in general sharp interface methods can be geometrically complex or otherwise difficult to implement in multiple spatial dimensions.

An alternative approach is the interface capturing method [18 21] which generally involves advecting a volume fraction or level set function. Analogous to shock capturing schemes developed to handle flow discontinuities in compressible flow algorithms, it can easily handle large topology changes in the interface. The method adds an additional conservation equation and is simple to implement in multiple dimensions but often requires numerical smearing of the material interface. In the presence of large density ratios and strong shocks this smearing can generate significant errors in computations of shock-interface interactions [22]. Methods for ensuring equilibrium conditions within the interface and maintaining its thickness throughout the simulation have been the motivation for an increasingly large body of work concerning two-phase compressible flows [19, 23, 28. One approach to counter numerical diffusion of the interface is implementation of high resolution WENO reconstruction [29, 30]. While the method can accurately capture material interfaces and has been successfully used to simulate the 
early stages of droplet breakup [8] without surface tension, the non-TVD nature of the WENO scheme may cause difficulties in computation of interface curvature, a required parameter for incorporating surface tension. When using the continuum surface force model, the interface curvature is commonly obtained by taking a second derivative of the volume fraction or, to prevent noise in the curvature, some other auxiliary function [31. Another option is to employ TVD spatial reconstruction with flux limiting and an interface compression technique shown to be successful in countering the numerical smearing of the interface. Such an approach was shown to accurately compute shock-interface interaction in the presence of high density ratios and strong shocks despite being discretely non-conservative 31. The interface compression scheme has since been further developed with non-linear preconditioning [22] and incorporated into physical timestepping with consistent thermodynamics 32 . However, the surface tension induced pressure jump across the interface has not yet been accounted for in the interface compression scheme.

The seven-equation non-equilibrium Baer-Nunziato model [33] is a frequent starting point for compressible multicomponent flow modeling. From there, it is common to assume pressure and velocity equilibrium among the fluid components which leads to a simpler five-equation model [21, 34]. The five equations consist of separate mass conservation equations for each phase, a quasi-conservative advection equation for the volume fraction, and a single momentum and energy equation for the total mixture. This assumption has been successfully applied to previous compressible models with capillary forces featuring all-speed relaxation [35] and exact [36] Riemann solvers that account for surface tension effects. Examples utilizing the HLLC Riemann solver include Le Martelot et al. 37] who developed a low speed model with surface tension for simulating nucleating boiling flows and Shukla 22 who demonstrated results with surface tension. Recently, a compressible framework utilizing the non-equilibrium seven equation Baer-Nunziato model [38] was developed with surface tension effects incorporated into a path-conservative Osher-type Riemann solver. Schmidmayer et al. 39] developed a compressible flow model with a conservative form of the surface tension force. However, specifically accounting for the surface tension induced pressure jump within the HLLC approximate Riemann solver remains to be discussed.

The objective of this work is to develop an efficient finite volume method for simulating high speed compressible flows with surface tension effects. Specifically, this includes developments to account for the surface tension induced pressure jump within the HLLC Riemann solver and the interface compression algorithm. Also, a simple and efficient method for computing interface curvature is developed and an acoustic non-dimensional scaling for the surface tension forces is proposed. For brevity, viscous forces are ignored and their inclusion will be the subject of future work.

The paper is organized as follows. Section 2 describes the model details including the governing equations, mixture rules, interface compression scheme, curvature computation, and the non-dimensionalization. Section 3 describes the spatial and temporal discretization of the overall method including the surface tension source terms and the interface compression scheme. Section 4 demonstrates the validity of the model by comparing numerical simulations with both analytical and experimental results. This is followed with a simulation to assess the ability of the approach to capture interfacial dynamics in a supersonic crossflow and conclusions are contained in Section 5 . 


\section{Governing equations}

The five equation model of Allaire [21] is employed in conjunction with capillary force terms written in a non-conservative form. As such, the compressible multicomponent Euler equations govern the flowfield [36:

$$
\begin{aligned}
& \frac{\partial \rho_{l} \phi_{l}}{\partial t}+\nabla \cdot\left(\rho_{l} \phi_{l} \mathbf{u}\right)=0, \\
& \frac{\partial \rho_{g} \phi_{g}}{\partial t}+\nabla \cdot\left(\rho_{g} \phi_{g} \mathbf{u}\right)=0, \\
& \frac{\partial \rho \mathbf{u}}{\partial t}+\nabla \cdot(\rho \mathbf{u u}+p \tilde{I})=\sigma \kappa \nabla \phi_{l}, \\
& \frac{\partial E}{\partial t}+\nabla \cdot((E+p) \mathbf{u})=\sigma \kappa \nabla \phi_{l} \cdot \mathbf{u}, \\
& \frac{\partial \phi_{l}}{\partial t}+\mathbf{u} \cdot \nabla \phi_{l}=0
\end{aligned}
$$

where $\rho_{l} \phi_{l}, \rho_{g} \phi_{g}$, and $\rho$ are the liquid, gas, and total densities, $\mathbf{u}=(u, v)^{T}$ is the velocity, $\phi_{l}$ is the liquid volume fraction, $p$ is the pressure, $\sigma$ is the surface tension coefficient, $\kappa$ is the interface curvature, and $E$ is the total energy

$$
E=\rho e+\frac{1}{2} \rho \mathbf{u} \cdot \mathbf{u}
$$

where $e$ is the specific internal energy. The fluid components are considered immiscible and the liquid and gas volume fraction functions $\left(\phi_{l}\right.$ and $\left.\phi_{g}\right)$ are used to capture the fluid interface. Mass is discretely conserved for each phase via individual mass conservation equations. Surface tension is implemented as a volume force as in the CSF model [3] with terms in both the momentum and energy equations [36. While a conservative form of the surface tension force exists [40, the present model utilizes the non-conservative form which enables flexible treatment of the curvature term $\kappa$ and its accuracy. Prior compressible flow models have employed both the conservative [22, 36] and non-conservative [35, 37, 38 model for surface tension.

\subsection{Mixture rules}

The diffuse interface capturing approach results in the material interface being spread over several grid cells. In this diffuse region, the fluid is treated as a mixture that is neither purely gas nor liquid. Flow variables within this mixture region are defined as follows. The gas $(g)$ and liquid $(l)$ volume fractions are related by

$$
\phi_{g}=1-\phi_{l} .
$$

The total density is given by

$$
\rho=\rho_{l} \phi_{l}+\rho_{g} \phi_{g}
$$

with internal energy

$$
\rho e=\rho_{l} \phi_{l} e_{l}+\rho_{g} \phi_{g} e_{g} .
$$

Utilizing an isobaric assumption [21, 30] and the stiffened gas equation of state (EOS) [41.

$$
p=(\gamma-1) \rho e-\gamma \pi_{\infty}
$$


the mixture EOS parameters $\Gamma$ and $\Pi$ are given by

$$
\begin{gathered}
\Gamma=\frac{1}{\gamma-1}=\Gamma_{g} \phi_{g}+\Gamma_{l} \phi_{l}, \\
\Pi=\frac{\gamma \pi_{\infty}}{\gamma-1}=\Pi_{g} \phi_{g}+\Pi_{l} \phi_{l},
\end{gathered}
$$

where $\Gamma_{g}=1 /\left(\gamma_{g}-1\right)$ and $\Pi_{g}=\gamma_{g} \pi_{\infty, g} /\left(\gamma_{g}-1\right)$ with $\Gamma_{l}$ and $\Pi_{l}$ defined similarly with the liquid parameters. The total energy can then be written as

$$
E=\Gamma p+\Pi+\frac{1}{2} \rho \mathbf{u} \cdot \mathbf{u}
$$

The isobaric assumption has been successfully employed in prior work to simulate surface tension in a compressible multicomponent framework [22, 35, 37]. Finally, the mixture speed of sound is given by

$$
c=\sqrt{\frac{\gamma\left(p+\pi_{\infty}\right)}{\rho}}
$$

where the mixture quantities $\gamma$ and $\pi_{\infty}$ are computed using Eqs. 7 and 8 . The mixture rules described above only apply in the diffuse interface region and simplify to either the gas or liquid parameters elsewhere.

\subsection{Surface tension induced pressure jump}

The eigensystem associated with Eqs. $1 \mathrm{a}, 1 \mathrm{e}$ is outlined in Appendix A. The eigenvalues are identical to the standard Euler system however surface tension terms appear in the eigenvectors. These terms result in a surface tension induced pressure jump across the contact wave $(*)$ given by (see [36])

$$
\Delta p_{*}=\sigma \kappa \Delta \phi_{l}
$$

This jump is consistent with the surface tension induced pressure jump described by the Young-Laplace law, i.e. see Figure 1 .

$$
\Delta p=\sigma \kappa .
$$

From Eq. 11, any jump in liquid volume fraction $\phi_{l}$ is associated with a corresponding pressure jump as a function of the curvature and surface tension coefficient. If the material interface is sharpened with an interface compression scheme, the liquid volume fraction distribution is modified near the interface and the corresponding pressure jump should also be modified according to Eq.11 to ensure the interface remains in equilibrium.

\subsection{Interface compression}

Material interfaces become smeared over time due to numerical diffusion. This can cause significant errors in the solution especially in the presence of shocks and rarefaction waves [22, 31. To counter this numerical smearing an interface compression technique is employed. The method numerically maintains the gas-liquid immiscibility condition 


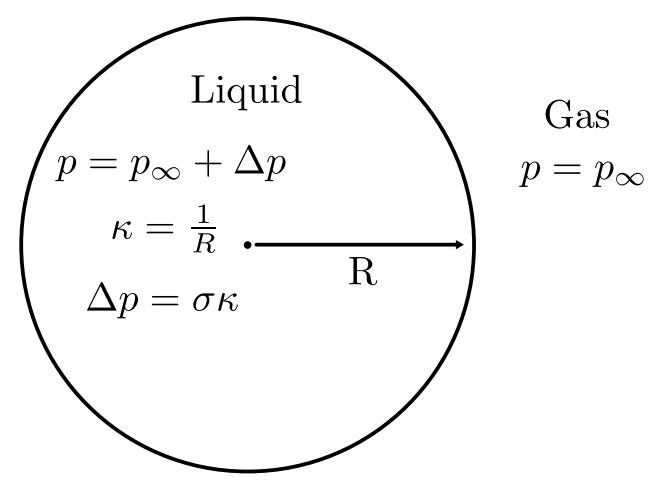

Figure 1: A liquid droplet in gas under ambient conditions experiences a pressure jump $\Delta p$ based on the surface tension coefficient $\sigma$ and droplet curvature $\kappa$. For the two dimensional case the curvature of a circular droplet is $1 / R$ where $R$ is the droplet radius.

by sharpening the material interface to only a few grid cells throughout the simulation. A form of the interface compression technique developed by Shukla et al. [31] with thermodynamically compatible extensions developed by Tiwari et al. 32 is employed in the present research with additional modifications to account for surface tension effects. With the method, a compression step in pseudo-time $\tau$ is performed after each physical timestep. Compression is performed by solving

$$
\begin{aligned}
\frac{\partial \rho_{l} \phi_{l}}{\partial \tau} & =\widehat{\mathcal{R}}_{l} \\
\frac{\partial \rho_{g} \phi_{g}}{\partial \tau} & =\widehat{\mathcal{R}}_{g} \\
\frac{\partial \rho \mathbf{u}}{\partial \tau} & =\mathbf{u} \widehat{\mathcal{R}} \\
\frac{\partial E}{\partial \tau} & =\kappa_{e} \widehat{\mathcal{R}}+\left(p\left(\Gamma_{l}-\Gamma_{g}\right)+\Pi_{l}-\Pi_{g}\right) \mathcal{R} \\
& +\sigma \kappa\left(\gamma_{l} \phi_{g}+\gamma_{g} \phi_{l}+\left(\gamma_{g}-\gamma_{l}\right) \Delta \tau \mathcal{R}-1\right) \Gamma_{l} \Gamma_{g} \mathcal{R} \\
\frac{\partial \phi_{l}}{\partial \tau} & =\mathcal{R}
\end{aligned}
$$

over the computational pseudo-timestep $\Delta \tau$ where $\kappa_{e}=\mathbf{u} \cdot \mathbf{u}$ and $\widehat{\mathcal{R}}=\widehat{\mathcal{R}}_{l}+\widehat{\mathcal{R}}_{g}$. The compression operators $\mathcal{R}, \widehat{\mathcal{R}}_{l}$, and $\widehat{\mathcal{R}}_{g}$ are defined as [31]:

$$
\begin{aligned}
\mathcal{R} & =\mathbf{n} \cdot\left[\nabla\left(\epsilon_{h}\left|\nabla \phi_{l}\right|\right)-\phi_{l}\left(1-\phi_{l}\right)\right], \\
\widehat{\mathcal{R}}_{l} & =H \mathbf{n} \cdot\left[\nabla\left(\epsilon_{h} \mathbf{n} \cdot \nabla\left(\rho_{l} \phi_{l}\right)\right)-\left(1-2 \phi_{l}\right) \nabla\left(\rho_{l} \phi_{l}\right)\right], \\
\widehat{\mathcal{R}}_{g} & =H \mathbf{n} \cdot\left[\nabla\left(\epsilon_{h} \mathbf{n} \cdot \nabla\left(\rho_{g} \phi_{g}\right)\right)-\left(1-2 \phi_{l}\right) \nabla\left(\rho_{g} \phi_{g}\right)\right]
\end{aligned}
$$

where the interface thickness is determined by the dissipation parameter $\epsilon_{h}$ which is set by the user. Following [22, $\epsilon_{h}=0.5 h$ and $0.72 h$ are employed for one and two dimensional simulations, respectively. Density variations can occur outside the interface region due to the compressible nature of the flow. The Heaviside function $H$ restricts 
the density compression to the interface region using [31]:

$$
H=\tanh \left[\left(\frac{\phi_{l}\left(1-\phi_{l}\right)}{10^{-2}}\right)^{2}\right]
$$

The second-order accurate centered finite volume spatial discretization described by Shukla et al. 31 is employed for the compression scheme and is outlined in Section 3.3 for completeness. Tiwari et al. 32] discuss the concept of consistent compression of the conservative variables. The present approach is unique to that of Shukla et al. [31] in that the conservative rather than the primitive variables are sharpened during pseudotime, while Tiwari et al. 32 also sharpen the conservative variables but do so inside the physical timestepping procedure.

Also unique to the present work is the $\sigma \kappa$ term in the energy compression equation (Eq. 13d). The term accounts for the surface tension effects and is derived by ensuring equilibrium conditions are maintained within the interface during a compression step. A static droplet with surface tension consists of a uniform pressure field $p$ with a surface tension induced pressure jump $\Delta p=\sigma \kappa \Delta \phi_{l}$ via Eq. 11. Using the equation of state the energy can be written in terms of the primitive variables as $E=\Gamma(p+\Delta p)+\Pi+\frac{1}{2} \rho\left(u^{2}+\right.$ $\left.v^{2}\right)$. For a static droplet in equilibrium $(u=v=0)$, this can be written as a function of the pseudo-time $\tau$ by:

$$
E(\tau)=\Gamma(\tau)\left(p+\sigma \kappa \phi_{l}(\tau)\right)+\Pi(\tau)
$$

where the mixture rules given by Eqs. 3,7 and 8 are also a function of the pseudo-time $\tau$ and are given by

$$
\begin{aligned}
\Gamma(\tau) & =\phi_{l}(\tau) \Gamma_{l}+\left(1-\phi_{l}(\tau)\right) \Gamma_{g} \\
\Pi(\tau) & =\phi_{l}(\tau) \Pi_{l}+\left(1-\phi_{l}(\tau)\right) \Pi_{g} .
\end{aligned}
$$

Assuming the interface remains in equilibrium after a compression timestep $\Delta \tau$, the resulting energy distribution is similarly given as

$$
E(\tau+\Delta \tau)=\Gamma(\tau+\Delta \tau)\left(p+\sigma \kappa \phi_{l}(\tau+\Delta \tau)\right)+\Pi(\tau+\Delta \tau) .
$$

Noting the volume fraction at the new pseudo-time is given by

$$
\phi_{l}(\tau+\Delta \tau)=\phi_{l}(\tau)+\Delta \tau \mathcal{R}
$$

it can be shown that Eq. 21, and therefore equilibrium conditions within the interface, will only hold if Eq. $13 \mathrm{~d}$ is used to perform the energy compression step.

To summarize, Eq. 13d ensures the pressure jump due to surface tension $(\Delta p=$ $\left.\sigma \kappa \Delta \phi_{l}\right)$ is sharpened in a manner consistent with the sharpened volume fraction so that the equilibrium in the interface is maintained. This behavior, and the effect of the surface tension compression term in Eq. 13d will be demonstrated numerically in Section 4.2. The interface compression scheme works in concert with the diffuse interface approach such that the interface remains sharp but with a mesh-representable profile. This allows gradients across the discontinuous interface to be calculated with reasonable accuracy. For example, to perform interface compression, the interface normals must first be computed. This procedure and the interface curvature calculation is discussed in the following section. 


\subsection{Curvature computation}

Interface curvature is calculated via the interface normals

$$
\kappa=-\nabla \cdot \mathbf{n}=-\nabla \cdot\left(\frac{\nabla \psi}{|\nabla \psi|}\right)
$$

where $\psi$ is a smoothed interface function [31]

$$
\psi=\frac{\phi_{l}^{\alpha}}{\phi_{l}^{\alpha}+\left(1-\phi_{l}\right)^{\alpha}}
$$

where $\alpha=0.1$. The vector quantity $\nabla \psi$ (and thus the normals) is calculated using fourth order central differences while the curvature itself, i.e.,

$$
\kappa=-\nabla \cdot \mathbf{n}
$$

is calculated using a second order central difference. This approach is found to improve convergence of the curvature calculation compared to utilizing second order differences for both the normal and curvature calculation.

The exact curvature for a two dimensional circular droplet is a constant $1 / R$ where $R$ is the radius of the droplet. For a droplet centered at $(0,0)$ the numerically computed curvature within the diffused interface region follows $1 / r$ where $r=\sqrt{x^{2}+y^{2}}$ is the local cell center value. This results in an imbalance between the pressure distribution and the surface tension force across the interface. To address this, the curvature value at $\phi_{l}=0.5$ (i.e. the actual interface location if the interface region were infinitely thin) should be extended outwards in both directions normal to the interface in a manner similar to one-sided ghost fluid extrapolation methods [42].

To achieve this, the present work utilizes a unique interfacial (as opposed to spatial) filtering strategy. The $n+1$ value of curvature at cell $i, j$ is corrected using weighted curvature values at iteration $n$ by:

$$
\kappa_{i, j}^{n+1}=\frac{\sum_{k=1}^{9} w_{k} \kappa_{k}^{n}}{\sum_{k=1}^{9} w_{k}}
$$

where $k$ refers to the $(i, j)$ index in the nine point stencil immediately surrounding the point $i, j$, i.e. $(i, j),(i+1, j),(i+1, j+1)$, etc. The weighting factor is similar to that employed by Renardy and Renardy [43] for their curvature interpolation and is given by

$$
w_{k}=\left(\phi_{l}\left(1-\phi_{l}\right)\right)_{k}^{2}
$$

which locally weights the curvature to the value at the center of the interface. The curvature is calculated and corrected at the end of each physical timestep before interface compression is performed. Figure 2(a) depicts the effect the filtering strategy has on correcting the curvature distribution near the interface. In essence, the filtering strategy drives the curvature distribution within the interface to the local value at $\phi_{l}=0.5$ which corresponds to the actual interface location if it were infinitely thin.

A grid convergence study is performed for the curvature calculation. The $L_{2}$ error defined by

$$
L_{2}=\sqrt{\frac{1}{N} \sum_{8}\left(\kappa_{i, j}-\frac{1}{R}\right)^{2}}
$$




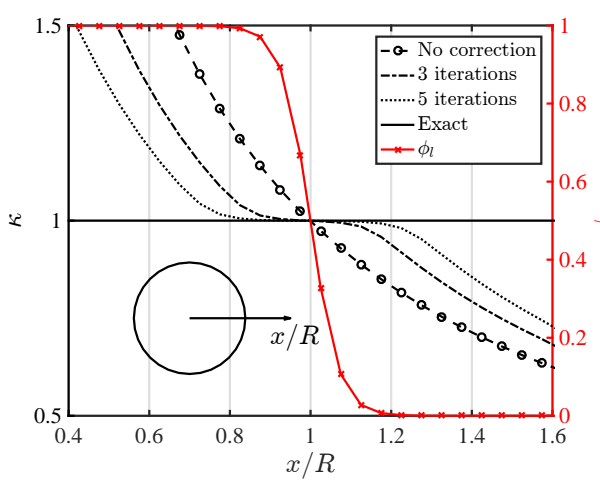

(a) Effect of correction iterations on curvature distribution.

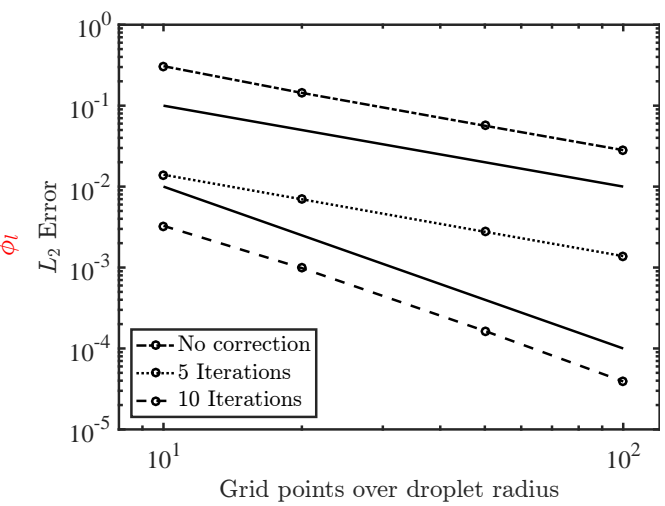

(b) Curvature $L_{2}$ error where the solid lines depict first and second order convergence.

Figure 2: Convergence of curvature distribution. No correction refers to the curvature calculated directly from Eq. 23

Table 1: Non-dimensional rules used in the model where primes indicate dimensional quantities and the subscript ' 0 ' represents the reference state.

\begin{tabular}{cc} 
Parameter & Rule \\
\hline Position & $x=x^{\prime} / l_{0}^{\prime}$ \\
Time & $t=t^{\prime} a_{0}^{\prime} / l_{0}^{\prime}$ \\
Velocity & $u=u^{\prime} / a_{0}^{\prime}$ \\
Density & $\rho=\rho^{\prime} / \rho_{0}^{\prime}$ \\
Pressure & $p=p^{\prime} / \rho_{0}^{\prime} a_{0}^{\prime 2}$ \\
Total Energy & $E=E^{\prime} / \rho_{0}^{\prime} a_{0}^{\prime 2}$ \\
Curvature & $\kappa=\kappa^{\prime} l_{0}^{\prime}$ \\
Surface tension coefficient & $\sigma=\frac{1}{\mathrm{We}_{a}}=\frac{\sigma_{0}^{\prime}}{\rho_{0}^{\prime} a_{0}^{\prime 2} l_{0}^{\prime}}$ \\
\hline
\end{tabular}

is computed where $\frac{1}{R}$ is the exact curvature and $\kappa_{i, j}$ is the numerically computed curvature in cell $i, j$. As the surface tension force (and curvature) is only applied in the diffuse interface region, $N$ is taken as the number of grid points within this region defined by the computational cells where $\phi_{l}\left(1-\phi_{l}\right)>0.001$. The results are depicted in Figure 2(b) and indicate that second order accuracy is obtained when ten correction iterations are applied. Five iterations shows a significant reduction in the error compared to the directly computed curvature but exhibits first order convergence. The effect of the correction iterations in practice can be seen in Section 4.3

\subsection{Final model and non-dimensionalization}

With the various ingredients of the overall model now specified, the governing equations are non-dimensionalized using the rules defined in Table 1 . Now, dimensional values are denoted with primes and the subscript ' 0 ' indicates a reference value. Substituting dimensional quantities using the rules in Table 1 into Eqs. 1a 1e results in the final 
non-dimensional model:

$$
\begin{gathered}
\frac{\partial \rho_{l} \phi_{l}}{\partial t}+\nabla \cdot\left(\rho_{l} \phi_{l} \mathbf{u}\right)=0 \\
\frac{\partial \rho_{g} \phi_{g}}{\partial t}+\nabla \cdot\left(\rho_{g} \phi_{g} \mathbf{u}\right)=0 \\
\frac{\partial \rho \mathbf{u}}{\partial t}+\nabla \cdot(\rho \mathbf{u u}+p \tilde{I})=\frac{1}{\mathrm{We}_{a}} \kappa \nabla \phi_{l}, \\
\frac{\partial E}{\partial t}+\nabla \cdot((E+p) \mathbf{u})=\frac{1}{\mathrm{We}_{a}} \kappa \nabla \phi_{l} \cdot \mathbf{u}, \\
\frac{\partial \phi_{l}}{\partial t}+\mathbf{u} \cdot \nabla \phi_{l}=0
\end{gathered}
$$

in conjunction with the stiffened gas EOS written in terms of the mixture rules (Eqs. 7 788:

$$
E=\Gamma p+\Pi+\frac{1}{2} \rho \mathbf{u} \cdot \mathbf{u} .
$$

Note that Eq. 29e is solved in a quasi-conservative form following Johnsen and Colonius [29]. The non-dimensionalization scales the surface tension force in the momentum and energy equations by an acoustic Weber number defined as:

$$
\mathrm{We}_{a}=\frac{\rho_{0}^{\prime} a_{0}^{\prime 2} l_{0}^{\prime}}{\sigma_{0}^{\prime}}
$$

where the primes indicate dimensional quantities and the subscript ' 0 ' represents the reference state with $\rho_{0}^{\prime}, a_{0}^{\prime}, l_{0}^{\prime}$, and $\sigma_{0}^{\prime}$ being the reference dimensional density, acoustic velocity, length scale, and surface tension coefficient, respectively. The acoustic Weber number is defined in the same manner that an acoustic Reynolds number [4] is created, where a natural reference speed for compressible flows is the speed of sound in a reference state.

\section{Numerical method}

The final model (Eqs. 29a 29e is discretized using the finite volume method on a uniform $(\Delta x=\Delta y=h)$ Cartesian grid. The resulting semi-discrete form of the equations is given by:

$$
\begin{aligned}
\frac{d_{i, j}}{d t}= & -\left(\frac{\mathbf{f}_{i+1 / 2}-\mathbf{f}_{i-1 / 2}}{\Delta x}\right) \\
& -\left(\frac{\mathbf{g}_{j+1 / 2}-\mathbf{g}_{j-1 / 2}}{\Delta y}\right)+\mathbf{S}_{i, j} \\
& =R\left({ }_{i, j}\right)
\end{aligned}
$$

where is the vector of state variables, $\mathbf{f}$ and $\mathbf{g}$ are the conservative convective fluxes in the $x$ and $y$ directions, $\mathbf{S}_{i, j}$ is the source term, and $R\left({ }_{i, j}\right)$ is the residual function. Spatial reconstruction is performed on the primitive variables using a TVD second order accurate scheme with the minmod limiter. 


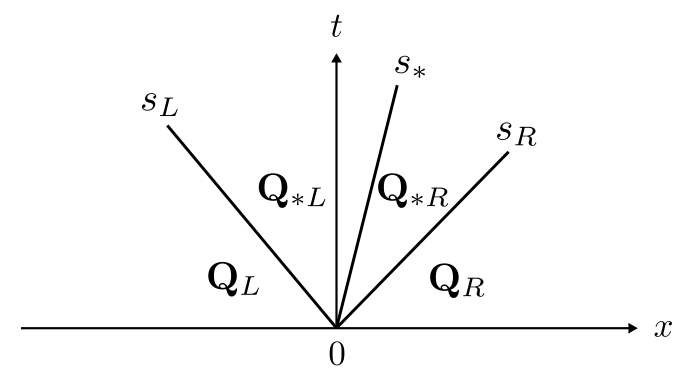

Figure 3: The Riemann fan for the HLLC Riemann solver considers two different states within the middle (*) region separated by a middle wave speed $s_{*}$, the contact wave. The left and right wave speeds are given by $s_{L}$ and $s_{R}$ respectively. The conserved variables in each region are denoted by $L, R, * L$, and $* R$.

The conserved variables are then integrated in time using the following third order TVD Runge-Kutta scheme [4]:

$$
\begin{aligned}
& { }_{i, j}^{(1)}={ }_{i, j}^{n}+\Delta t R\left(\begin{array}{l}
n \\
i, j
\end{array}\right), \\
& { }_{i, j}^{(2)}=\frac{3}{4}_{i, j}+\frac{1}{4}_{i, j}^{(1)}+\frac{1}{4} \Delta t R\left(_{i, j}^{(1)}\right), \\
& { }_{i, j}^{n+1}=\frac{1}{3}{ }_{i, j}+\frac{2}{3}{ }_{i, j}^{(2)}+\frac{2}{3} \Delta t R\left({ }_{i, j}^{(2)}\right) .
\end{aligned}
$$

\subsection{HLLC numerical flux and surface tension modifications}

The fluxes are upwinded using the HLLC approximate Riemann solver [46, 47]. To ensure oscillation free advection of material interfaces, the primitive variables are reconstructed and the HLLC features adaptions for a quasi-conservative form of the volume fraction transport equation [29. The present work includes several unique modifications to the HLLC solver to account for surface tension effects.

The HLLC solver restores the contact wave by considering two separate states in the star region (see Figure 3) such that the HLLC flux $\hat{\mathbf{f}}$ is defined by

$$
\hat{\mathbf{f}}= \begin{cases}\mathbf{f}_{\mathbf{L}} & \text { if } 0 \leq s_{L} \\ \mathbf{f}_{* \mathbf{L}} & \text { if } s_{L} \leq 0 \leq s_{*} \\ \mathbf{f}_{* \mathbf{R}} & \text { if } s_{*} \leq 0 \leq s_{R} \\ \mathbf{f}_{\mathbf{R}} & \text { if } 0 \geq s_{R} .\end{cases}
$$

The standard HLLC solver [46] without surface tension assumes a single pressure value $p_{*}$ across the contact discontinuity, i.e. $p_{*}=p_{* L}=p_{* R}$. The left and right states in the star region should be modified such that the pressure jump imposed by Eq. 11] is captured. Eq. 11 is written in terms of the right $R$ and left $L$ states of the Riemann problem as

$$
p_{* R}-p_{* L}=\sigma \kappa\left(\phi_{l, R}-\phi_{l, L}\right) .
$$

The present work accounts for this pressure jump by defining $p_{*}$ as:

$$
p_{*}=p_{* L}^{\prime}=p_{* R}^{\prime}
$$


where the primed left and right star region pressures are given by

$$
p_{* L}^{\prime}=p_{* L}-\sigma \kappa \phi_{l, L}, \quad p_{* R}^{\prime}=p_{* R}-\sigma \kappa \phi_{l, R}
$$

where $p_{* L}$ and $p_{* R}$ are defined as usual in HLLC:

$$
p_{* L}=p_{L}+\rho_{L}\left(s_{L}-u_{L}\right)\left(s_{*}-u_{L}\right), \quad p_{* R}=p_{R}+\rho_{R}\left(s_{R}-u_{R}\right)\left(s_{*}-u_{R}\right) .
$$

Using Eq. [36, the middle wave speed $s_{*}$ is now determined to be

$$
s_{*}=\frac{p_{R}-p_{L}+\rho_{L} u_{L}\left(s_{L}-u_{L}\right)-\rho_{R} u_{R}\left(s_{R}-u_{R}\right)-\sigma \kappa\left(\phi_{l, R}-\phi_{l, L}\right)}{\rho_{L}\left(s_{L}-u_{L}\right)-\rho_{R}\left(s_{R}-u_{R}\right)},
$$

which features a new term that accounts for the pressure jump across the interface due to surface tension. The final form of the HLLC flux is given compactly by [30]

$$
\hat{\mathbf{f}}=\frac{1+\operatorname{sgn}\left(s_{*}\right)}{2}\left[\mathbf{f}_{L}+s_{-}\left({ }_{* \mathbf{L}}-\mathbf{L}\right)\right]+\frac{1-\operatorname{sgn}\left(s_{*}\right)}{2}\left[\mathbf{f}_{R}+s_{+}\left(*_{\mathbf{R}}-\mathbf{R}\right)\right]
$$

in conjunction with the numerical flux $\mathbf{f}$ ( $\mathbf{g}$ for the $y$ direction) and state vector given by

$$
=\left(\begin{array}{c}
\rho_{l} \phi_{l} \\
\rho_{g} \phi_{g} \\
\rho u \\
\rho v \\
E \\
\phi_{l}
\end{array}\right), \quad \mathbf{f}=\left(\begin{array}{c}
\rho_{l} \phi_{l} u \\
\rho_{g} \phi_{g} u \\
\rho u u+p \\
\rho v u \\
(E+p) u \\
\phi_{l} u
\end{array}\right), \quad \mathbf{g}=\left(\begin{array}{c}
\rho_{l} \phi_{l} v \\
\rho_{g} \phi_{g} v \\
\rho u v \\
\rho v v+p \\
(E+p) v \\
\phi_{l} v
\end{array}\right),
$$

where the intermediate state is (for the $x$ direction)

$$
* \mathbf{K}=\frac{s_{K}-u_{K}}{s_{K}-s_{*}}\left(\begin{array}{c}
\left(\rho_{l} \phi_{l}\right)_{K} \\
\left(\rho_{g} \phi_{g}\right)_{K} \\
\rho_{K} s_{*} \\
\rho_{K} v_{K} \\
E_{K}+\left(s_{*}-u_{K}\right)\left(\rho_{K} s_{*}+\frac{p_{K}-\sigma \kappa \phi_{l, K}}{s_{K}-u_{K}}\right) \\
\phi_{l}
\end{array}\right)
$$

where $K=L, R$ refers to the left and right cell states and the curvature is defined here as $\kappa=\frac{1}{2}\left(\kappa_{L}+\kappa_{R}\right)$ where $\kappa_{L}$ and $\kappa_{R}$ are the curvatures defined at the cell center of the left and right cells, respectively. The wave speeds are computed using [48]

$$
s_{-}=\min \left(0, s_{L}\right), \quad s_{+}=\max \left(0, s_{R}\right)
$$

where

$$
s_{L}=\min \left(\bar{u}-\bar{c}, u_{L}-c_{L}\right), \quad s_{R}=\max \left(\bar{u}+\bar{c}, u_{L}+c_{L}\right)
$$

where, following [30], $\bar{u}=\frac{1}{2}\left(u_{L}+u_{R}\right)$ and $\bar{c}=\frac{1}{2}\left(c_{L}+c_{R}\right)$. Note that far from the interface $\left(\phi_{l, R}-\phi_{l, L}=0\right)$ or in the case of no surface tension $(\sigma=0)$ this choice of wave speeds is identical to that of Batten et al. [49] but now accounts for surface tension effects near the interface. Note that the HLLC is applied on a direction by direction basis and is straightforward to extend to multiple dimensions. 
Following Johnsen and Colonius [29] the advection equation is written in quasiconservative form

$$
\frac{\partial \phi_{l}}{\partial t}+\nabla \cdot\left(\phi_{l} \mathbf{u}\right)=\phi_{l} \nabla \cdot \mathbf{u}
$$

where the source term is evaluated within the $i, j$ computational cell using

$$
\left(\phi_{l} \nabla \cdot \mathbf{u}\right)_{i, j}=\left(\phi_{l}\right)_{i, j}\left[\frac{1}{\Delta x}\left(u_{i+\frac{1}{2}, j}-u_{i-\frac{1}{2}, j}\right)+\frac{1}{\Delta y}\left(v_{i, j+\frac{1}{2}}-v_{i, j-\frac{1}{2}}\right)\right] .
$$

The velocity values at the faces are appropriately upwinded by the HLLC solver, e.g. 29]

$$
u_{i-\frac{1}{2}, j}=\hat{u}\left({ }_{i-\frac{1}{2}, j}^{L},{ }_{i-\frac{1}{2}, j}^{R}\right)
$$

where

$$
\hat{u}=\frac{1+\operatorname{sgn}\left(s_{*}\right)}{2}\left[u_{L}+s_{-}\left(\frac{s_{L}-u_{L}}{s_{L}-s_{*}}-1\right)\right]+\frac{1-\operatorname{sgn}\left(s_{*}\right)}{2}\left[u_{R}+s_{+}\left(\frac{s_{R}-u_{R}}{s_{R}-s_{*}}-1\right)\right] .
$$

It should be noted that the advection equation is not a conservation law, so implementing it consistently into a Riemann solver is not immediately clear, as described by Johnsen and Colonius [29. As such, the discretization for Eqs. 46 48 from 29$]$ is a mathematically equivalent expression for the advection equation chosen specifically to adapt it to the HLLC Riemann solver while ensuring a discretization consistent with the energy equation and oscillation free advection of the interface. This approach is chosen for the present work due to its robustness as demonstrated in [29, 30], and 8], and its straightforward implementation in contact-preserving HLLC solvers.

\subsection{HLLC surface tension source terms}

The present work also utilizes the HLLC fluxes to evaluate the surface tension source terms. For the surface tension force in the $u$ momentum equation:

$$
\sigma \kappa \frac{\partial \phi}{\partial x}=\sigma \kappa_{i, j}\left[\frac{1}{\Delta x}\left(\phi_{i+\frac{1}{2}, j}-\phi_{i-\frac{1}{2}, j}\right)\right] .
$$

The force in the $v$ momentum equation is treated similarly with the face values of the volume fraction upwinded using the HLLC solver by

$$
\hat{\phi}=\frac{1+\operatorname{sgn}\left(s_{*}\right)}{2} \phi_{L}+\frac{1-\operatorname{sgn}\left(s_{*}\right)}{2} \phi_{R} .
$$

The surface tension term in the energy equation could be discretized with the cell center velocity by

$$
(\sigma \kappa \nabla \phi) \cdot \mathbf{u}=\sigma \kappa_{i, j}\left[\frac{u_{i, j}}{\Delta x}\left(\phi_{i+\frac{1}{2}, j}-\phi_{i-\frac{1}{2}, j}\right)+\frac{v_{i, j}}{\Delta y}\left(\phi_{i, j+\frac{1}{2}}-\phi_{i, j-\frac{1}{2}}\right)\right] .
$$

However, Coralic and Colonius [30, following [29, 36, noted that for consistency the velocity used in the source term and diffusive flux must be the same as that used in the advective numerical flux. This motivates the following decomposition of the surface 
tension source term in the energy equation, where, similarly to the advection equation, the term is first decomposed into conservative and non-conservative terms using

$$
\nabla \cdot(\phi \mathbf{u})=\phi \nabla \cdot \mathbf{u}+\nabla \phi \cdot \mathbf{u}
$$

such that

$$
\begin{aligned}
(\sigma \kappa \nabla \phi) \cdot \mathbf{u}= & \sigma \kappa[\nabla \cdot(\phi \mathbf{u})-\phi \nabla \cdot \mathbf{u}] \\
= & \sigma \kappa_{i, j}\left[\frac{1}{\Delta x}\left((\phi u)_{i+\frac{1}{2}, j}-(\phi u)_{i-\frac{1}{2}, j}\right)+\frac{1}{\Delta y}\left((\phi v)_{i, j+\frac{1}{2}}-(\phi v)_{i, j-\frac{1}{2}}\right)\right. \\
& \left.-\phi_{i, j}\left(\frac{1}{\Delta x}\left(u_{i+\frac{1}{2}, j}-u_{i-\frac{1}{2}, j}\right)+\frac{1}{\Delta y}\left(v_{i, j+\frac{1}{2}}-v_{i, j-\frac{1}{2}}\right)\right)\right]
\end{aligned}
$$

with

$$
(\phi u)_{i+\frac{1}{2}, j}=\phi_{i+\frac{1}{2}, j} u_{i+\frac{1}{2}, j}
$$

with the face values of $\phi$ and $u$ determined from the HLLC solver via Eqs. 50 and Eq. 48 . respectively. With this form, both the velocity and volume fraction in the source term are consistent with those used in the advective numerical flux.

\subsection{Compression discretization}

The compression operators $\mathcal{R}$ and $\widehat{\mathcal{R}}_{*}=\widehat{\mathcal{R}}_{l}, \widehat{\mathcal{R}}_{g}$ are discretized as in the work of Shukla et al. [31] for the $i, j$ computational cell as follows:

$$
\begin{aligned}
\mathcal{R} & =\left(\frac{f_{i+1 / 2, j}-f_{i-1 / 2, j}}{\Delta x}\right) n_{i, j}^{x}+\left(\frac{g_{i, j+1 / 2}-g_{i, j-1 / 2}}{\Delta y}\right) n_{i, j}^{y}, \\
\widehat{\mathcal{R}}_{*} & =H\left(\phi_{i, j}\right)\left[\left(\frac{f_{i+1 / 2, j}^{*}-f_{i-1 / 2, j}^{*}}{\Delta x}\right) n_{i, j}^{x}+\left(\frac{g_{i, j+1 / 2}^{*}-g_{i, j-1 / 2}^{*}}{\Delta y}\right) n_{i, j}^{y}\right. \\
& \left.-\left(1-2 \phi_{i, j}\right)\left(\frac{\left(\rho_{*} \phi_{*}\right)_{i+1, j}-\left(\rho_{*} \phi_{*}\right)_{i-1, j}}{2 \Delta x} n_{i, j}^{x}+\frac{\left(\rho_{*} \phi_{*}\right)_{i, j+1}-\left(\rho_{*} \phi_{*}\right)_{i, j-1}}{2 \Delta y} n_{i, j}^{y}\right)\right]
\end{aligned}
$$

where the subscript $l$ is dropped from the liquid volume fraction $\phi_{l}$ for convenience, $*=l, g$ denotes either the liquid or gas phasic densities, respectively, and $H\left(\phi_{i, j}\right)$ is the Heaviside function given by Eq. 17. The fluxes are given by

$$
\begin{aligned}
& f_{i-1 / 2, j}=\epsilon_{h}|\nabla \phi|_{i-1 / 2, j}-(\phi(1-\phi))_{i-1 / 2, j} \\
& f_{i-1 / 2, j}^{*}=\epsilon_{h} \mathbf{n}_{i-1 / 2, j} \cdot \nabla\left(\rho_{*} \phi_{*}\right)_{i-1 / 2, j}
\end{aligned}
$$

where

$$
\begin{aligned}
(\phi(1-\phi))_{i-1 / 2, j} & =\phi_{i-1 / 2, j}\left(1-\phi_{i-1 / 2, j}\right), \\
\phi_{i-1 / 2, j} & =\frac{\phi_{i, j}+\phi_{i-1, j}}{2} .
\end{aligned}
$$

The normal is computed by

$$
\mathbf{n}_{i-1 / 2, j}=\frac{\nabla \psi_{i-1 / 2, j}}{|\nabla \psi|_{i-1 / 2, j}}
$$


where $\psi$ is the smoothed interface function described in Eq. 24. The gradients of $\psi$ at the face are computed as

$$
\begin{aligned}
\left(\psi_{x}\right)_{i-1 / 2, j} & =\frac{\psi_{i, j}-\psi_{i-1, j}}{\Delta x}, \\
\left(\psi_{y}\right)_{i-1 / 2, j} & =\frac{\psi_{i, j+1}-\psi_{i, j-1}+\psi_{i-1, j+1}-\psi_{i-1, j-1}}{4 \Delta y},
\end{aligned}
$$

with gradients of $\left(\rho_{*} \phi_{*}\right)$ at the face calculated similarly. Finally, the gradient of the smoothed $\psi$ function is related to the volume fraction gradient by [31]:

$$
|\nabla \phi|_{i-1 / 2, j}=\frac{1}{\alpha}(\phi(1-\phi))_{i-1 / 2, j}^{1-\alpha}\left(\phi_{i-1 / 2, j}^{\alpha}+\left(1-\phi_{i-1 / 2, j}\right)^{\alpha}\right)^{2}|\nabla \psi|_{i-1 / 2, j} .
$$

The gradients and flux values for the other faces and directions are treated similarly.

\subsubsection{Compression timestepping}

In the present work, as in that of Shukla et al. 31, the compression algorithm is performed as an independent "post-processing" step after each physical timestep has completed. Note that the present method differs from that of [31] in two ways. First, compression is performed directly on the conservative variables based on the work of Tiwari et al. 32] rather than the primitive variables as in 31. The present compression scheme also differs in that the surface tension induced pressure jump across the interface is accounted for during the compression step. This compression step consists of a set of iterations advancing Eqs. 13a 13e in pseudo-time $\tau$ using the computational timestep $\Delta \tau$ until a steady state is reached and the desired interface thickness is recovered. For example, the $\phi_{l}$ compression equation (Eq. $13 \mathrm{e}$ ) is advanced in pseudo-time via

$$
\phi_{l}^{\tau+\Delta \tau}=\phi_{l}^{\tau}+\Delta \tau \mathcal{R}
$$

with the other compression equations treated similarly.

Note that Tiwari et al. 32] incorporates the compression step into the physical timestepping by scaling the compression operator $\mathcal{R}$ by a characteristic interface velocity:

$$
\mathcal{U}=4\left(\phi_{l}\left(1-\phi_{l}\right)|\mathbf{u}|\right)_{\max } .
$$

This essentially assumes that if the interface is not moving then numerical diffusion is minimal and interface compression is not needed. An analogous compression timestep for the present approach would be $\Delta \tau=\mathcal{U} \Delta t$ where $\Delta t$ is the physical timestep size employed. Shukla et al. 31] utilized a constant compression timestep of $\Delta \tau=0.2 \mathrm{~h}$ where $h$ is the uniform grid spacing. The current work utilizes a constant $\Delta \tau=0.1 h$ and performs between one and five compression iterations per physical timestep depending on the ratio of $\mathcal{U} \Delta t$ to $0.1 h$. This provides a good balance between computational effort, stability, and maintaining the desired interface thickness throughout the simulation.

\section{Results and discussion}

To verify the numerical model and implementation, one dimensional testing is performed first. This begins with a standard gas-liquid shock tube problem without surface 
tension. This is followed with two dimensional simulations of a circular droplet with surface tension to verify the ability of the model to capture correctly the surface tension induced pressure jump. Final verification is performed with spurious current and oscillating elliptical droplet tests. The method is then applied to Mach 1.47 and Mach 3 shock-droplet interaction problems.

\subsection{Gas-liquid Riemann problem}

To verify the solution algorithm is correctly implemented without surface tension, a gas-liquid Riemann problem previously used as a model for underwater explosions [19, 50] and verification of a multicomponent flow model [29, 30] is simulated. The problem includes highly compressed air and water at atmospheric pressure. The non-dimensional initial conditions are given by [50]:

$$
\left(\rho_{l} \phi_{l}, \rho_{g} \phi_{g}, u, p\right)= \begin{cases}\left(0,1.241\left(1-\phi_{l}\right), 0,2.753\right) & -1<x<0 \\ \left(0.991 \phi_{l}, 0,0,3.059 \times 10^{-4}\right) & 0 \leq x \leq 1\end{cases}
$$

with fluid properties $\gamma_{l}=5.5, \pi_{\infty, l}=1.505, \gamma_{g}=1.4, \pi_{\infty, g}=0$. Note the material interface is initialized in a slightly diffused state with an exponential function:

$$
\phi_{l}=1-\left(1+\exp \frac{x}{\epsilon_{h}}\right)^{-1}
$$

where $\epsilon_{h}=0.5 h, x$ is the cell center location and $h=0.0025$ is the grid spacing with 800 grid points. The results at a simulation time of $t=0.2$ and a constant timestep of $\Delta t=2.5 \times 10^{-4}$ are shown in Figure 4 and compare well to the exact solution. The interface compression scheme successfully maintains the sharp material interface throughout the simulation.

\subsection{Static droplet with exact curvature}

The following simulation illustrates the ability of the present model to capture the surface tension induced pressure jump exactly if exact curvature is prescribed and shows the effect of the surface tension term in the energy compression equation. Without this term, spurious oscillations are introduced into the solution even for a simple static droplet with exact prescribed curvature. The simulation corresponds to a unity radius water droplet in $\operatorname{air}\left(\gamma_{g}=1.4, \pi_{\infty, g}=0\right)$ with density ratio 1000. Following Shukla et al. [31, the non-dimensional EOS parameters for the water are given by $\gamma_{l}=4.4$ and $\pi_{\infty, l}=6000$ using the reference state $\rho_{0}^{\prime}=1 \mathrm{~kg} / \mathrm{m}^{3}$ and $a_{0}^{\prime}=\sqrt{10^{5}} \mathrm{~m} / \mathrm{s}$. A unity acoustic Weber number is set such that the surface tension induced pressure jump across the interface is dictated by Eq. 11. or in terms of the $\phi_{l}$ distribution, $\Delta p=\frac{\kappa}{\mathrm{We}_{a}} \phi_{l}=\phi_{l}$. The initial conditions are:

$$
\left(\rho_{l} \phi_{l}, \rho_{g} \phi_{g}, u, v, p\right)=\left(1000 \phi_{l},\left(1-\phi_{l}\right), 0,0,1+\Delta p\right) .
$$

To demonstrate the effect of compression, the interface is initialized in an overly diffused state with $\Delta=2$ via:

$$
\phi_{l}=\left(\begin{array}{c}
\left.1+\exp \frac{r-1}{\Delta \epsilon_{h}}\right)^{-1} \\
16
\end{array}\right.
$$




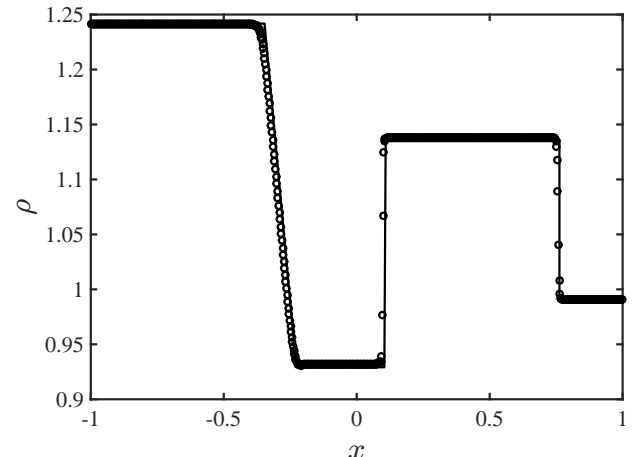

(a) Density $\rho$

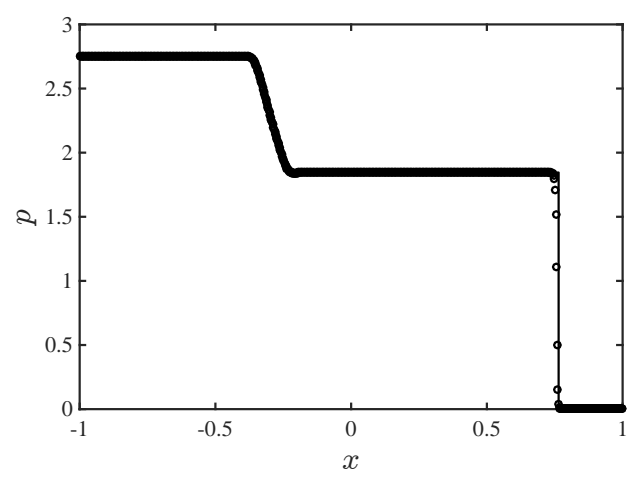

(c) Pressure $p$

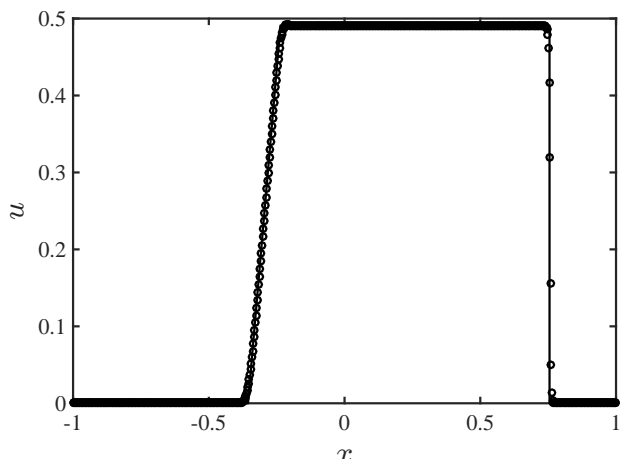

(b) Velocity $u$

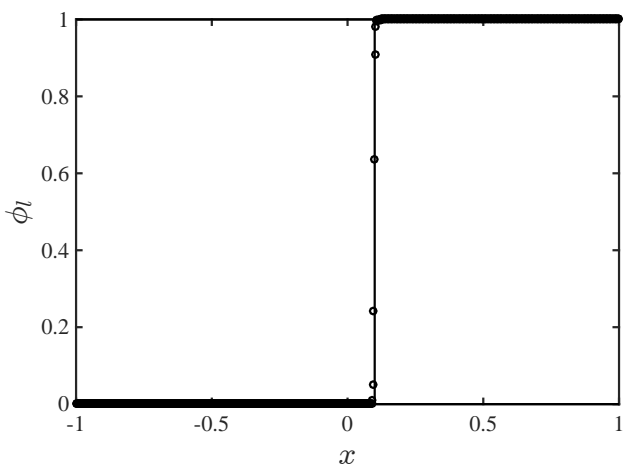

(d) Volume fraction $\phi_{l}$

Figure 4: Data (o) at $t=0.2$ using 800 grid points compared to the exact solution $(-)$. 


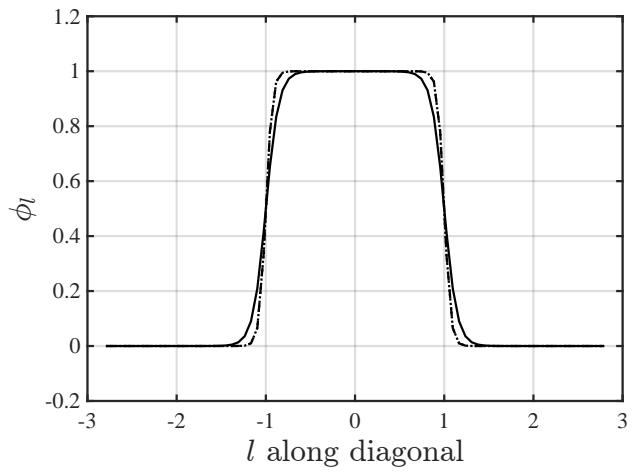

(a) Volume fraction $\phi_{l}$

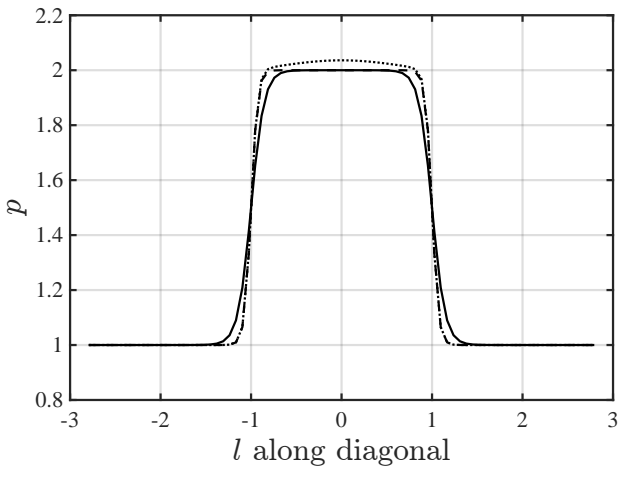

(b) Pressure $p$

Figure 5: The volume fraction $\phi_{l}$ (a) and pressure (b) is plotted over a diagonal cut from $(x, y)=(-2,-2)$ to $(2,2)$. Lines depict the diffused initial conditions $(-)$ and results at the final time with $(--)$ and without $(. \cdot)$ the surface tension term included in the energy compression equation.

Table $2: L_{\infty}$ norms of the pressure and velocity error for the simulation with exact curvature with and without the surface tension term included in the energy compression equation.

\begin{tabular}{c|cc} 
& \multicolumn{2}{|c}{ Surface tension compression term } \\
Included & Not included \\
\hline Error & $2.12 \times 10^{-11}$ & $3.60 \times 10^{-2}$ \\
Velocity $(\max |\mathbf{u}|)$ & $2.49 \times 10^{-14}$ & $1.55 \times 10^{-5}$
\end{tabular}

with $r=\sqrt{x^{2}+y^{2}}$ and $\epsilon_{h}=0.72 h$. Extrapolation boundary conditions are employed on a mesh with $80 \times 80$ grid points $(h=0.05)$ for a $[-2,2] \times[-2,2]$ domain. The solution is computed using a CFL condition of 0.6 to a final time of $t=10$.

Plots of the results along a diagonal cut from $(x, y)=(-2,-2)$ to $(2,2)$ are depicted in Figure 5. The surface tension compression term appears only in the energy compression equation and does not affect the behavior of the compression scheme on the volume fraction distribution, as shown in Figure $5(\mathrm{a})$. The pressure distributions with and without the surface tension compression term are shown in Figure 5(b). As the curvature is forced to the exact value, the pressure jump should correspond directly to the jump in volume fraction as dictated by Eq. 11 .

Table 2 compares the $L_{\infty}$ norm of the error in the pressure and velocity distributions for the simulations. Since the droplet is initially static, the velocity field should remain zero if the pressure and surface tension forces are in equilibrium throughout the simulation. The spurious pressure oscillations observed if surface tension is not accounted for in the interface compression scheme are found to generate disturbances in the velocity field. The pressure error is measured by subtracting the ambient pressure and the exact pressure jump (according to Eq. 11) from the computed pressure. The results indicate that if the exact curvature is provided, the present model maintains equilibrium across the interface and captures the surface tension induced pressure jump to within machine precision. 


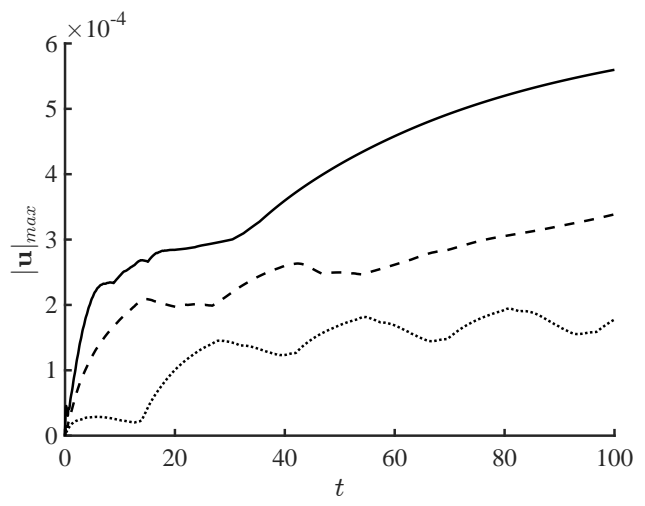

(a) Five correction iterations

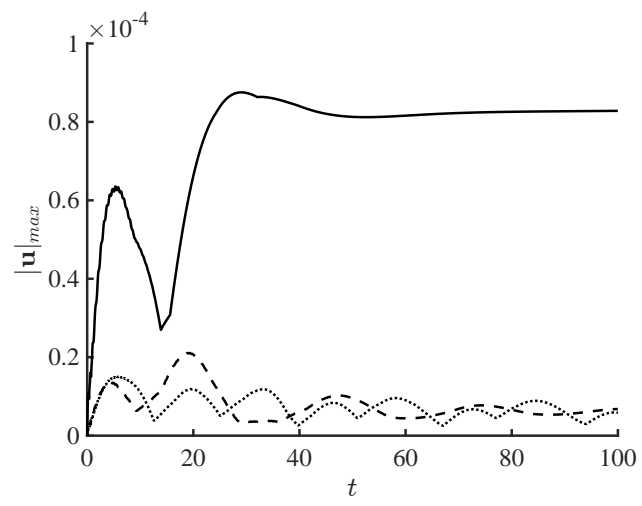

(b) Ten correction iterations

Figure 6: Maximum velocity as a function of time for a $40 \times 40(-), 80 \times 80(--)$, and $160 \times 160(. \cdot)$ grid with five (a) and ten (b) curvature correction iterations.

\subsection{Parasitic currents}

Errors in the calculation of the interface curvature can increase the magnitude of unphysical "parasitic currents" inherent in surface tension simulations [36, 51, 52. As the current work does not include physical diffusion (i.e. $R e \rightarrow \infty$, but numerical diffusion exists in practice), the effect of the parasitic currents could potentially be detrimental and should be investigated.

A simple test is performed to check if the currents distort the material interface. The same initial conditions as the previous test case are employed but without the overly diffused interface initialization, i.e. $\Delta=1$ in Eq. 71 which corresponds to the interface width enforced by the solution of the interface compression algorithm at steady state. The droplet should remain stationary and the interface should not become distorted due to the parasitic currents. The curvature is calculated numerically using the procedure in Section 2.4 with five and ten correction iterations. The solution is advanced to nondimensional time $t=100$ using a CFL of 0.6 .

Typically the effect of the parasitic currents are reported in terms of the Laplace number $L a=\rho \sigma D / \mu^{2}$ and the capillary number measured as $C a=|\mathbf{u}|_{\max } \mu / \sigma$ (for example see [53, 54]). Since the current work does not include physical diffusion terms, these parameters are undefined. Instead, the maximum velocity during the spurious current test is depicted in Figure 6 for several grid resolutions. When five correction iterations (Figure 6(a) are employed the currents gradually increase over time. With ten iterations (Figure $6(\mathrm{~b})$ ), the currents oscillate with time but the mean value remains almost constant. In both cases the maximum velocity is shown to decrease with mesh refinement. In all cases the currents remain small (on the order of $10^{-4}-10^{-5}$ ) and no signs of interface distortion are observed in any of the performed simulations. As physical diffusion terms are presently neglected and should damp the spurious currents, this result is satisfactory. 


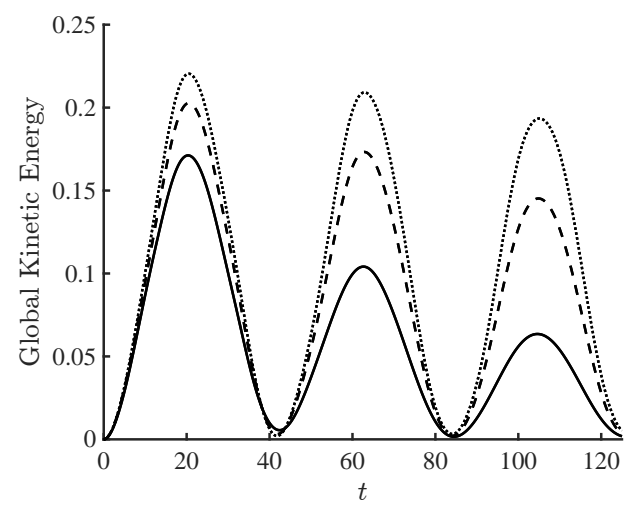

Figure 7: Global kinetic energy for an oscillating elliptical droplet on a $100 \times 100(-), 200 \times 200(--)$, and $400 \times 400(. \cdot)$ grid for a surface tension coefficient of $\sigma=1$ and liquid density of $\rho_{l}=1000$.

\subsection{Oscillating ellipse}

An oscillating ellipse study is frequently performed to validate the accuracy of surface tension implementations [22, 36, 55, 56] and ensure the model is capable of predicting physical solutions. The period of oscillation can be computed analytically [57, 58]:

$$
T=2 \pi \sqrt{\frac{\left(\rho_{l}+\rho_{g}\right) R^{3}}{6 \sigma}}
$$

where $T$ is the oscillation period, $\sigma$ is the surface tension coefficient, $\rho_{l}$ and $\rho_{g}$ are the liquid and gas densities, and $R$ is the equivalent circular radius. Following Shukla [22] who simulated a similar problem, all units are non-dimensional. An ellipse defined by

$$
1=\frac{x^{2}}{a^{2}}+\frac{y^{2}}{b^{2}}
$$

has an equivalent circular radius of $R=\sqrt{a b}$ assuming mass is conserved. Several oscillating ellipse simulations using $a=5 / 4$ and $b=4 / 5$ were performed with a gas density of $\rho_{g}=1$ and liquid densities of $\rho_{l}=100$ and 1000 with varying surface tension coefficients of $\sigma=10 / 3,1$, and $1 / 4$. The domain and material EOS properties are the same as in the parasitic currents test above. All simulations were performed with a CFL of 0.6 with five curvature correction iterations. The numerical oscillation period was measured using the variation in global kinetic energy $\left(\frac{1}{2} \int \rho|\mathbf{u}|^{2} d x d y\right)$ where a full period occurs every other trough. Figure 7 shows the behavior of the global kinetic energy over time for several grid resolutions where a gradual decay is observed due to numerical dissipation. Overall, excellent agreement was found with the analytical result, as shown in Figure 8 for a $200 \times 200$ grid.

\section{5. $M_{s}=1.47$ shock-droplet interaction}

To further validate the present model, a simulation was performed for comparison with the experimental breakup visualizations of a $4 \mathrm{~mm}$ thick cylindrical water column in a crossflow by Igra and Takayama [59]. The cylindrical water column geometry can be 


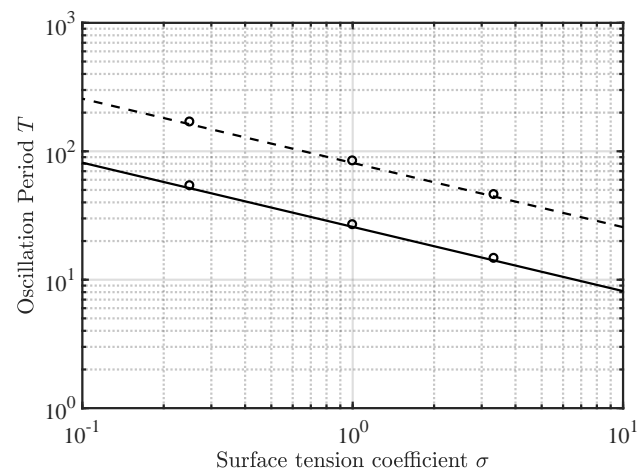

Figure 8: Analytical results for liquid densities of $\rho_{l}=100(-)$ and $\rho_{l}=1000(--)$ compared to the computed (o) ellipse oscillation period as a function of the surface tension coefficient.

modeled with a two-dimensional domain and has been studied previously by a number of researchers to examine the early stages of the shock-droplet interaction and deformation [7, 8, 13, 14, 22, 31, 60, 61.

The problem consists of a $M_{s}=1.47$ shockwave in air $\left(\rho_{g}=1.2 \mathrm{~kg} / \mathrm{m}^{3}\right)$ impacting a water column $\left(\rho_{l}=1000 \mathrm{~kg} / \mathrm{m}^{3}\right)$ with a nominal diameter of $D=4.8 \mathrm{~mm}$ at its centerline. The acoustic Weber number is set such that the crossflow Weber number matches the experimental value of 6900 [59]. Molecular diffusion effects are neglected in the present simulation since the experimental Reynolds number is very large at $\mathrm{Re}=$ $1.12 \times 10^{5}$. The EOS parameters employed are the same as the previous simulations with $\gamma_{g}=1.4, \pi_{\infty, g}=0$ for the gas and $\gamma_{l}=4.4, \pi_{\infty, l}=6 \times 10^{8} \mathrm{~Pa}$ for the liquid. The solution domain extends from $[-7.5 D, 7.5 D] \times[0,7.5 D]$ with a Dirichlet boundary upstream, a symmetry condition along $y_{\min }$, and extrapolation boundaries elsewhere. Uniform grid spacing is used with a grid resolution of $D / 200$. This results in a domain consisting of $3000 \times 1500$ grid points. The incident shock is initialized one droplet diameter upstream from the leading edge of the droplet.

A time history of the simulation in comparison to the experimental holographic interferograms of Igra and Takayama [59] is shown in Figure 9. A similar approach to that described by Meng and Colonius [8] is used to match the simulation times to the experiment by measuring the time and distance traveled by the incident shockwave in Figures $9(\mathrm{a})$ and $9(\mathrm{~b})$ This provided a time scaling which accurately determined the simulation times for comparing the remaining experimental images. It was assumed the actual location of the gas-liquid interface lies at the center of the dark shadows in the experimental images. It was noted by Igra and Takayama [59] that these dark rings indicate wet areas where the water column is in contact with the glass surface of the test section.

The incident and reflected shock structures show good qualitative agreement with the experiment in Figures 9(a) 9(d). The crossflow induced by the passage of the shockwave begins to gradually deform the water column and leads to the appearance of a recirculation region behind the trailing edge of the droplet. Similar behavior is observed and discussed in more detail by Meng and Colonius [8]. Figure 10 depicts the later stages of the deformation process where the general shape of the water column is still generally dis- 


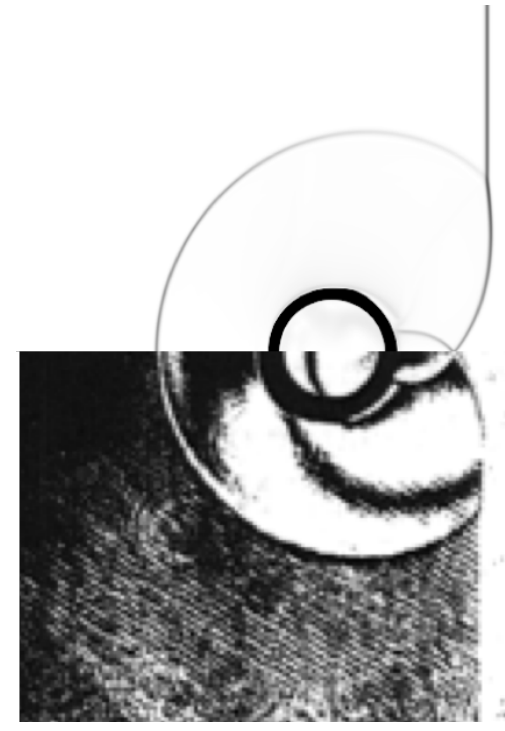

(a) $t=26.5 \mu \mathrm{s}$

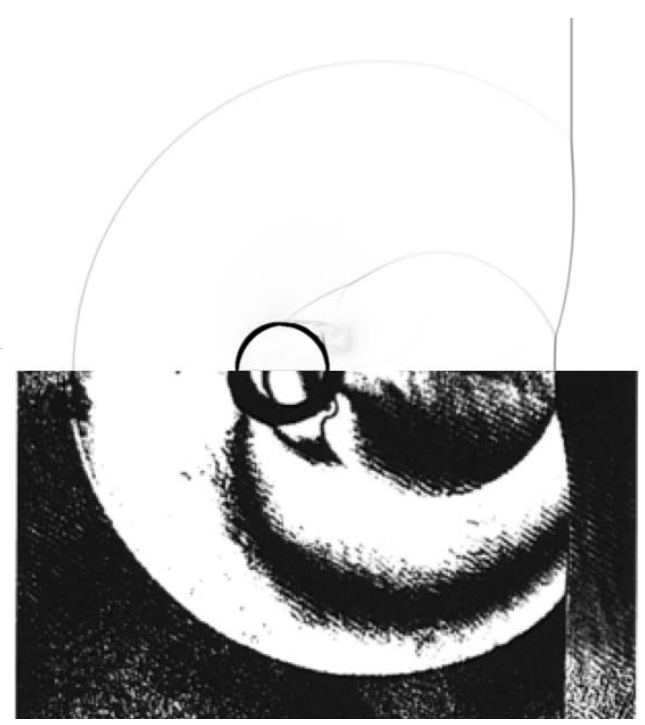

(b) $t=44 \mu \mathrm{s}$

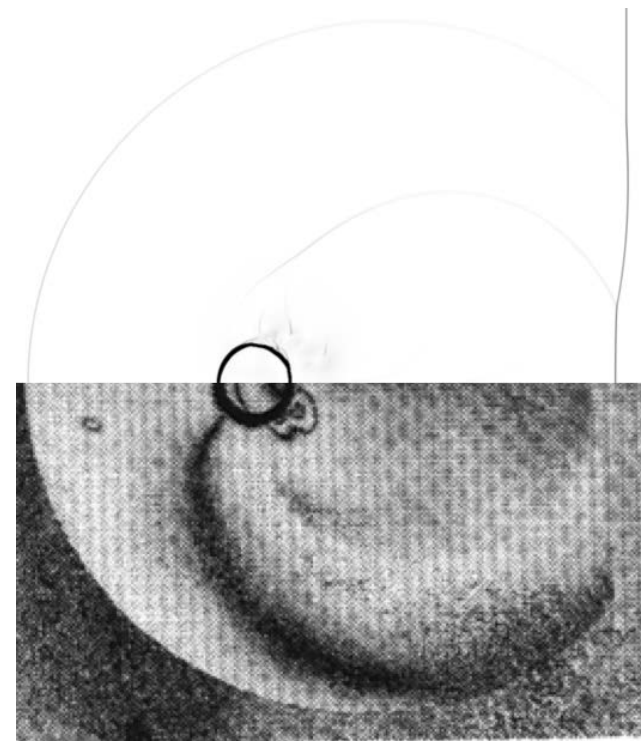

(c) $t=61.4 \mu \mathrm{s}$

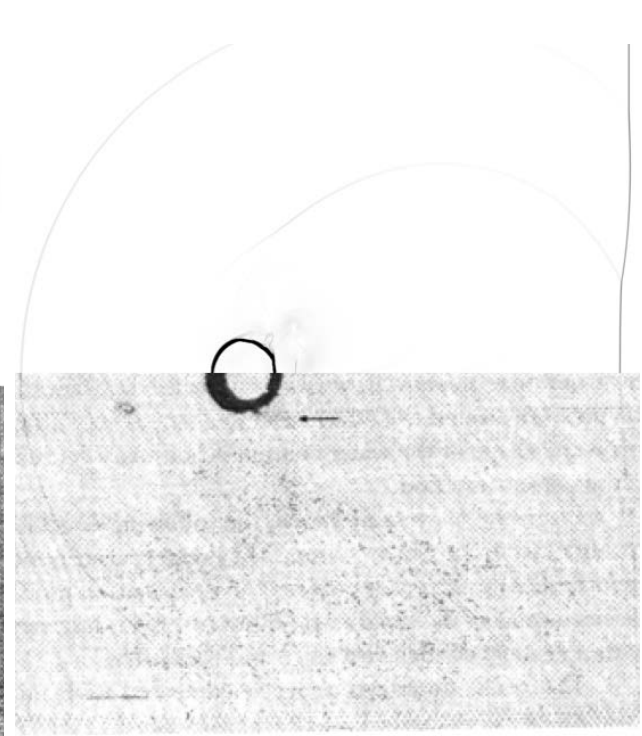

(d) $t=70.1 \mu \mathrm{s}$

Figure 9: Time history of two-dimensional shock-droplet interaction and breakup. The top half of each image depicts numerical Schlieren. The bottom half shows holographic interferograms from the experiment of Igra and Takayama [59]. 


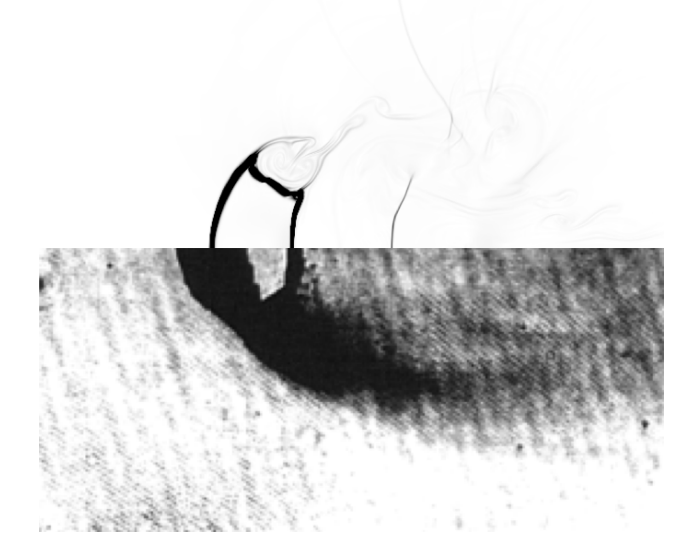

Figure 10: Numerical Schlieren of simulation (top) vs. holographic interferogram from experiment 59 (bottom) at $t=209 \mu \mathrm{s}$.

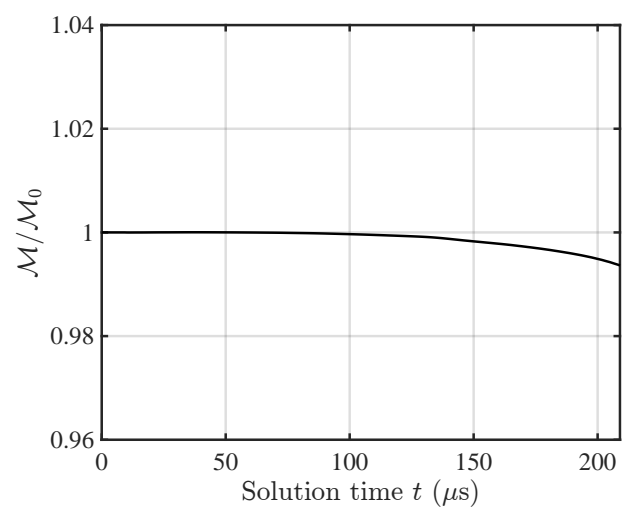

Figure 11: Plot of integrated liquid mass fraction $\left(\mathcal{M}=\int \rho_{l} \phi_{l} d V\right)$ over time.

cernible in the experimental image and indicates significant deformation has begun [59. Overall, good qualitative agreement is observed compared to the numerical result which maintains the thickness of the interface throughout the simulation. This property allows straightforward visualization of the deformation of the column over time.

Note that the presently employed interface compression operators are discretely nonconservative, as discussed in more detail in 31. As a measure of the conservation of the scheme, Figure 11 plots the ratio of the integrated liquid mass over the initial value for the present simulation. As indicated in the figure, by the end of the simulation less than one percent of the total liquid mass is lost due to the interface compression scheme at this resolution.

\section{6. $M_{s}=3$ shock-droplet interaction}

To demonstrate the stability and behavior of the method with a lower Weber number and in a supersonic flow, a shockwave traveling in air at a Mach number $M_{s}=3$ is 
simulated impacting a water column with unity radius. The initial conditions correspond to those in 62 but non-dimensionalized by the column radius $r=1.75 \mathrm{~mm}$. The domain is $[-5,10] \times[-5,5]$ and extrapolation boundary conditions are applied on all four boundaries. At the end of the simulation the upstream inlet conditions are verified to be unchanged from the initial conditions which are given in non-dimensional form by

$$
\left(\rho_{l} \phi_{l}, \rho_{g} \phi_{g}, u, v, p\right)= \begin{cases}(0,3.857,2.629,0,10.333) & x \leq-2 / 1.75 \\ \left(1000 \phi_{l},\left(1-\phi_{l}\right), 0,0,1+\Delta p\right) & \text { otherwise }\end{cases}
$$

where $\gamma_{l}=4.4, \pi_{\infty, l}=6000, \gamma_{g}=1.4$ and $\pi_{\infty, g}=0$ and

$$
\phi_{l}=\left(1+\exp \frac{r-1}{\epsilon_{h}}\right)^{-1}
$$

with $r=\sqrt{x^{2}+y^{2}}$ and $\epsilon_{h}=0.72 h$. The same CFL condition and EOS properties of the previous simulations are employed with five curvature correction iterations. The acoustic Weber number is set to $\mathrm{We}_{a}=1.446$ making the initial pressure jump distribution $\Delta p=$ $\phi_{l} / 1.446$. The corresponding crossflow Weber number is $\mathrm{We}=77$ based on the post-shock flow conditions. Note that these conditions correspond to a supersonic crossflow $(M=$ 1.36) which generates a leading bow shock and a variation in local flow conditions over the droplet. Liquid atomization in supersonic flow has seen significantly less investigation than subsonic flows. To provide a basis for comparison to subsonic flow conditions an effective Weber number can be computed behind the bow shock using the crossflow Mach and Weber numbers and the velocity and density normal shock relations [17, i.e.

$$
\mathrm{We}_{\mathrm{eff}}=\frac{2+(\gamma-1) M^{2}}{(\gamma+1) M^{2}} \mathrm{We}=47.7
$$

In essence, this considers a stream-line emanating upstream from the stagnation point of the droplet to estimate the jump conditions across the bow shock using normal shock relations.

Figure 12 depicts a numerical Schlieren (exponentially spaced normalized density gradient) of the early stages of the Euler simulation characterized by the incident shockwave impacting the water column. This results in a transmitted shockwave inside the column and a reflected bow shock upstream. The effects of surface tension are not significant at this stage. The results agree well with the previous AUSM-based simulation by Chang and Liou 62. This includes a slight but expected variation in the transmitted shockwave location in the water column at later times due to the difference in the EOS parameters employed for the liquid phase.

The effects with vs. without surface tension are examined in Figure 13 in terms of the overall flow structure. In the simulation without surface tension, increased grid resolution leads to the development of interfacial instabilities which tend to grow over time. These features are especially noticeable on the top and bottom of the water column and appear to generate local recirculation regions observed at $t=12$. Similar flow features are not observed in the case with surface tension which provides a restoring force that resists high interface curvature. This test case demonstrates the approach can capture interface dynamics in a supersonic crossflow. 


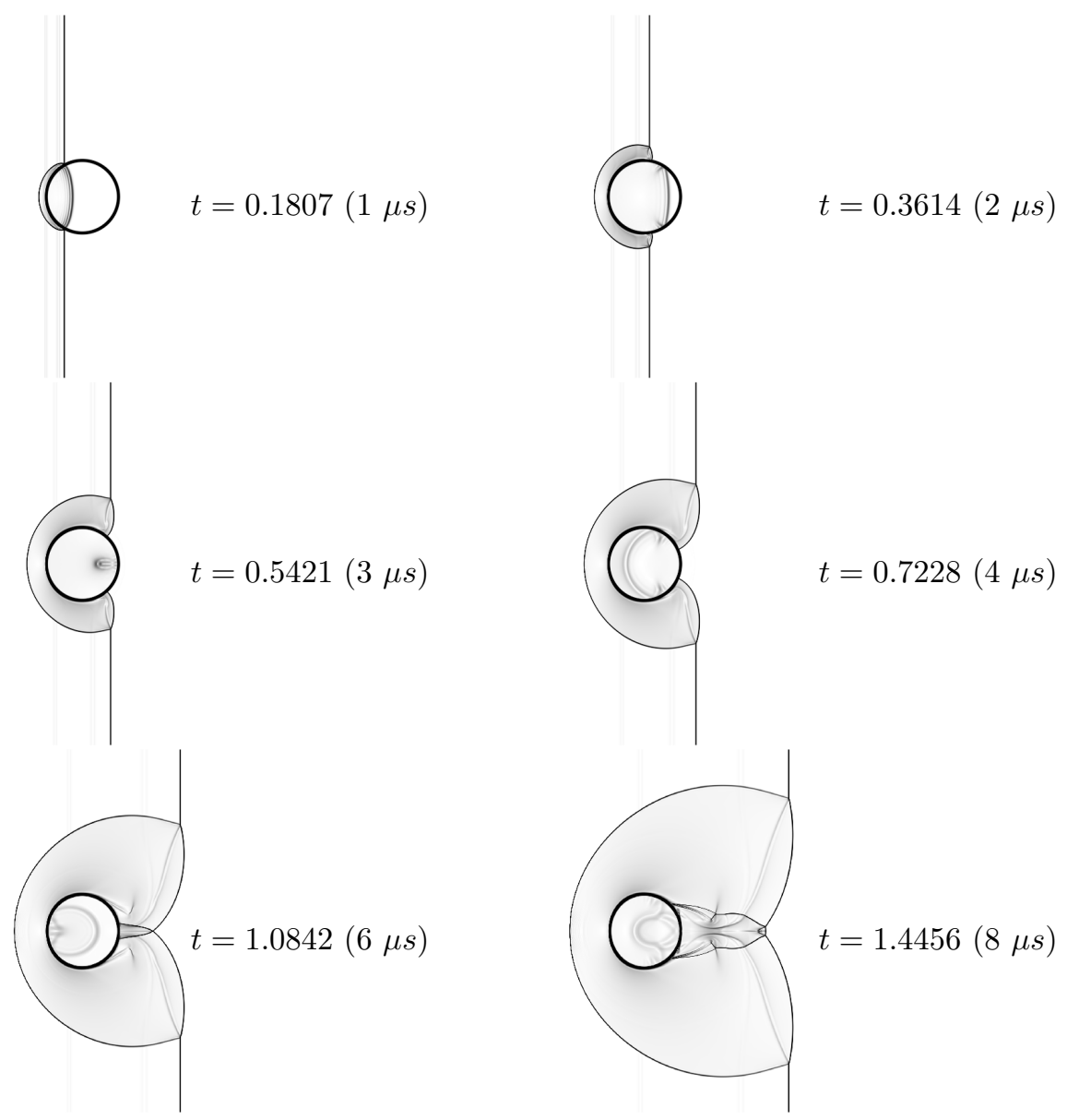

Figure 12: Numerical schlieren of the early stages of droplet shock interaction on a $2400 \times 1600$ grid for non-dimensional times of $0.1807,0.3614,0.5421,0.7228,1.0842$, and 1.4456 . Based on the droplet dimensions in 62, these correspond to physical times of $1,2,3,4,6$, and $8 \mu \mathrm{s}$. 

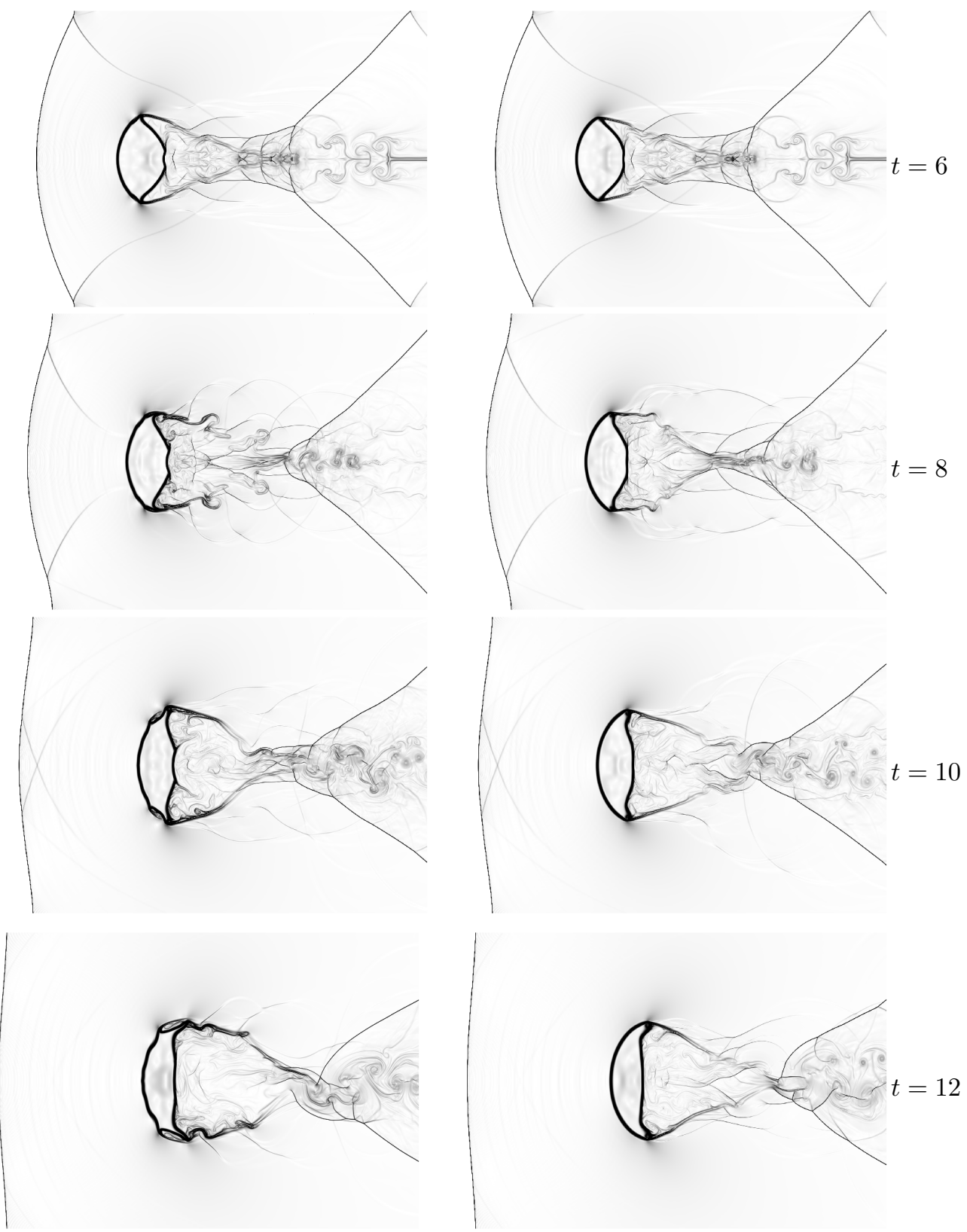

(a) Without surface tension

(b) With surface tension

Figure 13: Numerical schlieren of later stages of simulations without surface tension (a) vs. with surface tension (b) at non-dimensional times of $6,8,10,12$. Based on the droplet dimensions in 62], these correspond to physical times of $33.2,44.2,55.3,66.4 \mu \mathrm{s}$. 


\subsection{Secondary atomization in Mach 0.5 crossflow}

Validation of the method in capturing the various secondary breakup modes is performed for We $=5-100$. The breakup behavior is dependent on the Weber number with vibrational, bag, and multimode regimes 2. The initial conditions correspond to a static liquid droplet with non-dimensional diameter $D=1$ and initial density ratio $\epsilon=\rho_{l} / \rho_{g}=10$ interacting with a shockwave traveling at $M_{s}=1.39$. The EOS parameters are chosen to be $\gamma_{l}=4.4, \pi_{\infty, l}=60, \gamma_{g}=1.4$ and $\pi_{\infty, g}=0$. The characteristic breakup time scales with the density ratio $T=t u /(D \sqrt{\epsilon})$ 63. and the low liquid density was chosen only to reduce the computational cost of the simulations similar to prior studies 64, 65. Additionally, the EOS parameters were chosen so that the liquid is essentially incompressible while accounting for the lower density. The overall domain consists of $4800 \times 1920$ grid points (320 grid points across the initial droplet diameter) spanning 12 and 30 droplet radii in the transverse and stream wise directions, respectively. Only half of the physical domain was simulated by placing the droplet centroid at the y-minimum axis with a symmetry boundary condition enforced.

Figure 14 shows a table of the droplet breakup behavior for the range of crossflow Weber numbers simulated. The post-shock airstream is traveling at a Mach number of 0.5 and the resulting aerodynamic forces acting on the initially stationary droplet cause it to gradually deform. As expected, for $\mathrm{We}<10$, the droplet responds in a vibrational mode where the droplet either remains a single droplet or breaks up into a couple of individual droplets. As the Weber number increases it moves into bag and then multimode type breakup behavior where the droplets stretch out into long ligaments before eventually breaking up into small droplets. The results have good general agreement with the experimentally categorized behavior and have good qualitative agreement with prior low density ratio two dimensional simulations [64, 65.

\section{Conclusion}

A finite volume method is developed to simulate compressible multicomponent flow with capillary forces. An extension of the HLLC Riemann solver and an interface compression scheme are developed to account for the pressure jump across an interface from surface tension. If the exact curvature is known, the method is shown to capture the surface tension induced pressure jump to within machine precision for a static droplet. Additionally, a simple method for calculating the interface curvature is developed. In the case of a circular droplet, the interface curvature is shown to be second order accurate with sufficient correction iterations. Parasitic currents are found not to adversely affect the interface despite molecular diffusion terms being neglected at this time. The overall method is further verified through comparison to the analytical relationship of an oscillating elliptical droplet. A $M_{s}=1.47$ shock-droplet interaction shows good qualitative agreement with experimental visualizations. A $M_{s}=3$ simulation is performed to demonstrate the capability of the method to capture droplet deformation in a supersonic cross flow. The general characteristics of secondary atomization at a range of Weber numbers are also captured in a series of simulations. The method was developed and tested in two dimensions, but the extension to three dimensions is straightforward. 


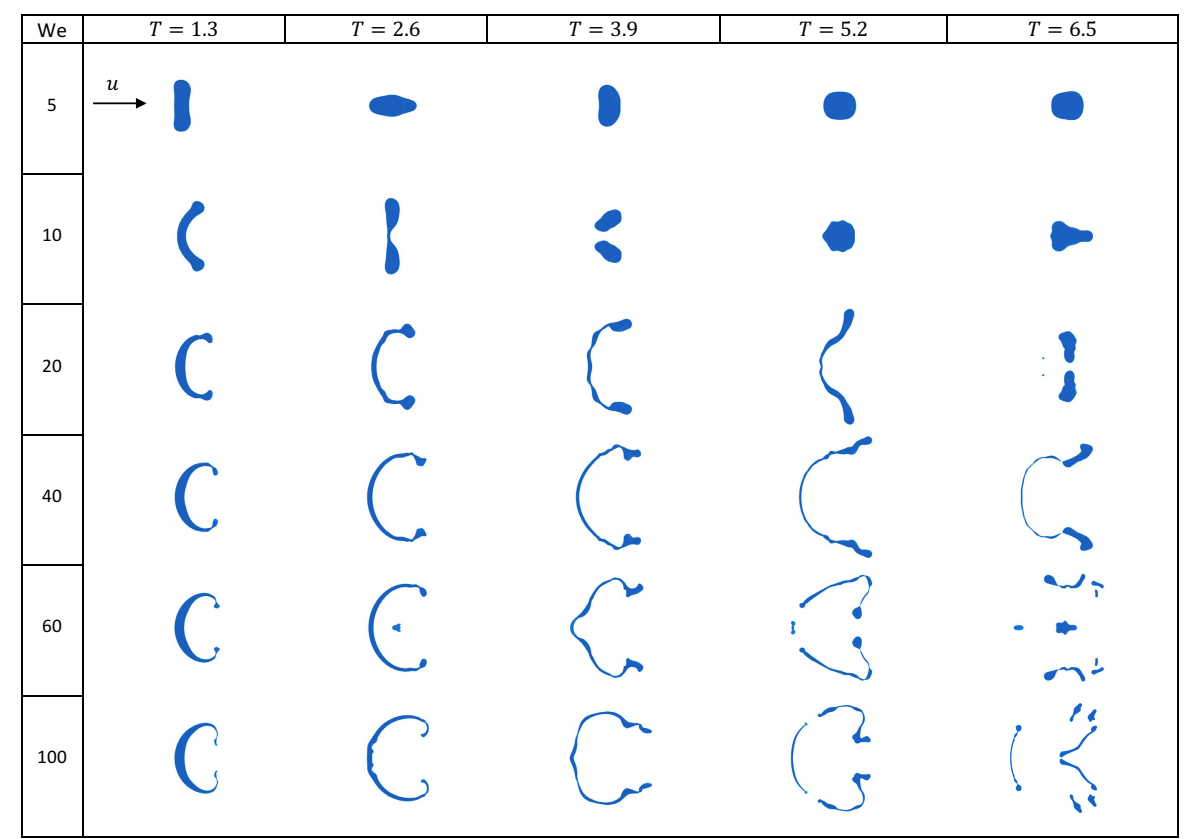

Figure 14: Droplet breakup evolution for different Weber numbers in a Mach 0.5 crossflow. The non-dimensional characteristic time $T=t u /(D \sqrt{\epsilon})[63$ is computed using the post shock conditions.

\section{Acknowledgments}

This work is supported by Taitech, Inc. under sub-contract TS15-16-02-004 (primary contract FA8650-14-D-2316). The research reported in this paper is partially supported by the HPC@ISU equipment at Iowa State University, some of which has been purchased through funding provided by NSF under MRI grant number CNS 1229081 and CRI grant number 1205413. The authors would also like to thank the anonymous reviewers for their constructive comments.

\section{Appendix A: Eigenvalues and Eigenvectors}

In dimensional form the state vector, fluxes, and source terms are defined as

$$
=\left(\begin{array}{c}
\rho_{l} \phi_{l} \\
\rho_{g} \phi_{g} \\
\rho u \\
\rho v \\
E \\
\phi_{l}
\end{array}\right), \quad \mathbf{f}()=\left(\begin{array}{c}
\rho_{l} \phi_{l} u \\
\rho_{g} \phi_{g} u \\
\rho u u+p \\
\rho v u \\
(E+p) u \\
0
\end{array}\right), \quad \mathbf{g}()=\left(\begin{array}{c}
\rho_{l} \phi_{l} v \\
\rho_{g} \phi_{g} v \\
\rho u v \\
\rho v v+p \\
(E+p) v \\
0
\end{array}\right)
$$




$$
\mathbf{S}_{\mathbf{x}}=\left(\begin{array}{cccccc}
0 & 0 & 0 & 0 & 0 & 0 \\
0 & 0 & 0 & 0 & 0 & 0 \\
0 & 0 & 0 & 0 & 0 & \sigma \kappa \\
0 & 0 & 0 & 0 & 0 & 0 \\
0 & 0 & 0 & 0 & 0 & \sigma \kappa u \\
0 & 0 & 0 & 0 & 0 & u
\end{array}\right), \quad \mathbf{S}_{\mathbf{y}}=\left(\begin{array}{cccccc}
0 & 0 & 0 & 0 & 0 & 0 \\
0 & 0 & 0 & 0 & 0 & 0 \\
0 & 0 & 0 & 0 & 0 & 0 \\
0 & 0 & 0 & 0 & 0 & \sigma \kappa \\
0 & 0 & 0 & 0 & 0 & \sigma \kappa v \\
0 & 0 & 0 & 0 & 0 & v
\end{array}\right),
$$

where a compact matrix-vector form of the governing equations (Eqs. 1a.1e) is

$$
\frac{\partial}{\partial t}+\frac{\partial \mathbf{f}()}{\partial x}+\frac{\partial \mathbf{g}()}{\partial y}=\mathbf{S}_{\mathbf{x}} \frac{\partial}{\partial x}+\mathbf{S}_{\mathbf{y}} \frac{\partial}{\partial y}
$$

Assuming spatially and temporally frozen curvature [36], this system can be written in terms of the primitive variables $\mathbf{V}=\left(\rho_{l} \phi_{l}, \rho_{g} \phi_{g}, u, v, p, \phi_{l}\right)^{T}$ in quasi-linear form as

$$
\frac{\partial \mathbf{V}}{\partial t}+\mathbf{A}(\mathbf{V}) \frac{\partial \mathbf{V}}{\partial x}+\mathbf{B}(\mathbf{V}) \frac{\partial \mathbf{V}}{\partial y}=0
$$

where the Jacobian matrices are defined as

$$
\begin{aligned}
\mathbf{A}(\mathbf{V}) & =\frac{\partial \mathbf{V}}{\partial}\left(\frac{\partial \mathbf{f}()}{\partial}-\mathbf{S}_{\mathbf{x}}\right) \frac{\partial}{\partial \mathbf{V}} \\
\mathbf{B}(\mathbf{V}) & =\frac{\partial \mathbf{V}}{\partial}\left(\frac{\partial \mathbf{g}()}{\partial}-\mathbf{S}_{\mathbf{y}}\right) \frac{\partial}{\partial \mathbf{V}} \\
\frac{\partial}{\partial \mathbf{V}} & =\left(\frac{\partial \mathbf{V}}{\partial}\right)^{-1} .
\end{aligned}
$$

The HLLC solver is applied on a direction by direction basis so the conditions along the $x$ direction are examined noting that conditions along the $y$ direction can be similarly derived. The eigenvalues $\boldsymbol{\Lambda}_{\mathbf{A}}$ and right eigenvector $\mathbf{A}$ associated with the Jacobian $\mathbf{A}=$ ${ }_{\mathbf{A}} \boldsymbol{\Lambda}_{\mathbf{A} \mathbf{A}}{ }^{-1}$ are:

$$
\mathbf{A}=\left(\begin{array}{cccccc}
\frac{-\rho_{l} \phi_{l}}{c} & 1 & 0 & 0 & 0 & \frac{\rho_{l} \phi_{l}}{c} \\
\frac{-\rho_{g} \phi_{g}}{c} & 0 & 1 & 0 & 0 & \frac{\rho_{g} \phi_{g}}{c} \\
1 & 0 & 0 & 0 & 0 & 1 \\
0 & 0 & 0 & 1 & 0 & 0 \\
-\rho c & 0 & 0 & 0 & \sigma \kappa & \rho c \\
0 & 0 & 0 & 0 & 1 & 0
\end{array}\right), \quad \boldsymbol{\Lambda}_{\mathbf{A}}=\left(\begin{array}{cccccc}
u-c & 0 & 0 & 0 & 0 & 0 \\
0 & u & 0 & 0 & 0 & 0 \\
0 & 0 & u & 0 & 0 & 0 \\
0 & 0 & 0 & u & 0 & 0 \\
0 & 0 & 0 & 0 & u & 0 \\
0 & 0 & 0 & 0 & 0 & u+c
\end{array}\right) .
$$

The eigenvalues are identical to the standard Euler system without surface tension and with a full set of eigenvectors the model is hyperbolic. Note surface tension terms do appear in the eigenvectors which account for the surface tension induced pressure jump across the material contact denoted by Eq. 35 .

\section{References}

[1] J. Lee, K.-C. Lin, D. Eklund, Challenges in Fuel Injection for High-Speed Propulsion Systems, AIAA Journal 53 (2015) 1405-1423.

[2] D. R. Guildenbecher, C. López-Rivera, P. E. Sojka, Secondary atomization, Experiments in Fluids 46 (2009) 371-402. 
[3] J. U. Brackbill, D. Kothe, C. Zemach, A Continuum Method for Modeling Surface Tension, Journal of Computational Physics 100 (1992) 335-354.

[4] D. Gueyffier, J. Li, A. Nadim, R. Scardovelli, S. Zaleski, Volume-of-Fluid Interface Tracking with Smoothed Surface Stress Methods for Three-Dimensional Flows, Journal of Computational Physics 152 (1999) 423-456.

[5] S. Popinet, S. Zaleski, A Front-Tracking Algorithm for Accurate Representation of Surface Tension, International Journal for Numerical Methods in Fluids 30 (1999) 775-793.

[6] R. Scardovelli, S. Zaleski, Direct Numerical Simulation of Free-Surface and Interfacial Flow, Annual Review of Fluid Mechanics 31 (1999) 567-603.

[7] H. Chen, Two-Dimensional Simulation of Stripping Breakup of a Water Droplet, AIAA Journal 46 (2008) 1135-1143.

[8] J. C. Meng, T. Colonius, Numerical simulations of the early stages of high-speed droplet breakup, Shock Waves (2014) 399-414.

[9] M. Gorokhovski, M. Herrmann, Modeling Primary Atomization, Annual Review of Fluid Mechanics 40 (2008) 343-366.

[10] S. O. Unverdi, G. Tryggvason, A front-tracking method for viscous, incompressible, multi-fluid flows, Journal of Computational Physics 100 (1992) 25-37.

[11] G. Tryggvason, B. Bunner, A. Esmaeeli, D. Juric, N. Al-Rawahi, W. Tauber, J. Han, S. Nas, Y. J. Jan, A front-tracking method for the computations of multiphase flow, Journal of Computational Physics 169 (2001) 708-759.

[12] R. Abgrall, S. Karni, Computations of compressible multifluids, Journal of Computational Physics 169 (2001) 594-623.

[13] H. Terashima, G. Tryggvason, A front-tracking/ghost-fluid method for fluid interfaces in compressible flows, Journal of Computational Physics 228 (2009) 4012-4037.

[14] H. Terashima, G. Tryggvason, A front-tracking method with projected interface conditions for compressible multi-fluid flows, Computers and Fluids 39 (2010) 1804-1814.

[15] H. Luo, J. D. Baum, R. Lohner, On the computation of multi-material flows using ALE formulation, Journal of Computational Physics 194 (2004) 304-328.

[16] C. H. Chang, X. Deng, T. G. Theofanous, Direct numerical simulation of interfacial instabilities: A consistent, conservative, all-speed, sharp-interface method, Journal of Computational Physics 242 (2013) 946-990.

[17] F. Xiao, Z. G. Wang, M. B. Sun, J. H. Liang, N. Liu, Large eddy simulation of liquid jet primary breakup in supersonic air crossflow, International Journal of Multiphase Flow 87 (2016) 229-240.

[18] C. W. Hirt, B. D. Nichols, Volume of fluid (VOF) method for the dynamics of free boundaries, Journal of Computational Physics 39 (1981) 201-225.

[19] K.-M. Shyue, An Efficient Shock-Capturing Algorithm for Compressible Multicomponent Problems, Journal of Computational Physics 142 (1998) 208-242.

[20] R. Saurel, R. Abgrall, A Simple Method for Compressible Multifluid Flows, SIAM Journal on Scientific Computing 21 (1999) 1115-1145.

[21] G. Allaire, S. Clerc, S. Kokh, A Five-Equation Model for the Simulation of Interfaces between Compressible Fluids, Journal of Computational Physics 181 (2002) 577-616.

[22] R. K. Shukla, Nonlinear preconditioning for efficient and accurate interface capturing in simulation of multicomponent compressible flows, Journal of Computational Physics 276 (2014) 508-540.

[23] K. K. So, X. Y. Hu, N. A. Adams, Anti-diffusion interface sharpening technique for two-phase compressible flow simulations, Journal of Computational Physics 231 (2012) 4304-4323.

[24] S. Alahyari Beig, E. Johnsen, Maintaining interface equilibrium conditions in compressible multiphase flows using interface capturing, Journal of Computational Physics 302 (2015) 548-566.

[25] K. M. Shyue, F. Xiao, An Eulerian interface sharpening algorithm for compressible two-phase flow: The algebraic THINC approach, Journal of Computational Physics 268 (2014) 326-354.

[26] R. Abgrall, How to prevent pressure oscillations in multicomponent flow calculations: A Quasi Conservative Approach, Journal of Computational Physics (1996) 150-160.

[27] E. Johnsen, F. Ham, Preventing numerical errors generated by interface-capturing schemes in compressible multi-material flows, Journal of Computational Physics 231 (2012) 5705-5717.

[28] P. Movahed, E. Johnsen, A solution-adaptive method for efficient compressible multifluid simulations, with application to the Richtmyer-Meshkov instability, Journal of Computational Physics 239 (2013) 166-186.

[29] E. Johnsen, T. Colonius, Implementation of WENO schemes in compressible multicomponent flow problems, Journal of Computational Physics 219 (2006) 715-732.

[30] V. Coralic, T. Colonius, Finite-volume WENO scheme for viscous compressible multicomponent 
flows, Journal of Computational Physics 274 (2014) 95-121.

[31] R. K. Shukla, C. Pantano, J. B. Freund, An interface capturing method for the simulation of multi-phase compressible flows, Journal of Computational Physics 229 (2010) 7411-7439.

[32] A. Tiwari, J. B. Freund, C. Pantano, A diffuse interface model with immiscibility preservation, Journal of Computational Physics 252 (2013) 290-309.

[33] M. R. Baer, J. W. Nunziato, A two-phase mixture theory for the deflagration-to-detonation transition (ddt) in reactive granular materials, International Journal of Multiphase Flow 12 (1986) 861-889.

[34] A. K. Kapila, R. Menikoff, J. B. Bdzil, S. F. Son, D. S. Stewart, Two-phase modeling of deflagrationto-detonation transition in granular materials: Reduced equations, Physics of Fluids 13 (2001) 3002-3024.

[35] B. Braconnier, B. Nkonga, An all-speed relaxation scheme for interface flows with surface tension, Journal of Computational Physics 228 (2009) 5722-5739.

[36] G. Perigaud, R. Saurel, A compressible flow model with capillary effects, Journal of Computational Physics 209 (2005) 139-178.

[37] S. Le Martelot, R. Saurel, B. Nkonga, Towards the direct numerical simulation of nucleate boiling flows, International Journal of Multiphase Flow 66 (2014) 62-78.

[38] N. T. Nguyen, M. Dumbser, A path-conservative finite volume scheme for compressible multi-phase flows with surface tension, Applied Mathematics and Computation 271 (2015) 959-978.

[39] K. Schmidmayer, F. Petitpas, E. Daniel, N. Favrie, S. Gavrilyuk, A model and numerical method for compressible flows with capillary effects, Journal of Computational Physics 334 (2017) 468-496.

[40] D. Gueyffier, J. Li, A. Nadim, R. Scardovelli, S. Zaleski, Volume-of-Fluid Interface Tracking with Smoothed Surface Stress Methods for Three-Dimensional Flows, Journal of Computational Physics 152 (1999) 423-456.

[41] F. Harlow, A. Amsden, Fluid Dynamics, Technical Report LA-4700, Los Alamos National Laboratory, Los Alamos, NM, 1971.

[42] T. D. Aslam, A partial differential equation approach to multidimensional extrapolation, Journal of Computational Physics 193 (2004) 349-355.

[43] Y. Renardy, M. Renardy, PROST: A Parabolic Reconstruction of Surface Tension for the Volumeof-Fluid Method, Journal of Computational Physics 183 (2002) 400-421.

[44] J. D. Regele, D. R. Kassoy, M. Aslani, O. V. Vasilyev, Evolution of detonation formation initiated by a spatially distributed, transient energy source, Journal of Fluid Mechanics 802 (2016) 305-332.

[45] S. Gottlieb, C.-W. Shu, Total variation diminishing Runge-Kutta schemes, Mathematics of Computation 67 (1998) 73-85.

[46] E. F. Toro, M. Spruce, W. Speares, Restoration of the contact surface in the HLL-Riemann solver, Shock Waves 4 (1994) 25-34.

[47] E. F. Toro, Riemann Solvers and Numerical Methods for Fluid Dynamics: A Practical Introduction, Springer, New York, 2009.

[48] B. Einfeldt, C. D. Munz, P. L. Roe, B. Sjögreen, On Godunov-type methods near low densities, Journal of Computational Physics 92 (1991) 273-295.

[49] P. Batten, N. Clarke, C. Lambert, D. M. Causon, On the Choice of Wavespeeds for the HLLC Riemann Solver, SIAM Journal on Scientific Computing 18 (1997) 1553-1570.

[50] J. P. Cocchi, R. Saurel, J. C. Loraud, Treatment of interface problems with Godunov-type schemes, Shock Waves 5 (1996) 347-357.

[51] D. J. E. Harvie, M. R. Davidson, M. Rudman, An analysis of parasitic current generation in Volume of Fluid simulations, Applied Mathematical Modelling 30 (2006) 1056-1066.

[52] B. Lafaurie, C. Nardone, R. Scardovelli, S. Zaleski, G. Zanetti, Modelling Merging and Fragmentation in Multiphase Flows with SURFER, 1994.

[53] J. Luo, X. Y. Hu, N. A. Adams, A conservative sharp interface method for incompressible multiphase flows, Journal of Computational Physics 284 (2015) 547-565.

[54] M. Owkes, O. Desjardins, A mesh-decoupled height function method for computing interface curvature, Journal of Computational Physics 281 (2015) 285-300.

[55] S. Ii, S. Kazuyasu, S. Takeuchi, S. Takagi, Y. Matsumoto, F. Xiao, An interface capturing method with a continuous function: The THINC method with multi-dimensional reconstruction, Journal of Computational Physics 231 (2012) 2328-2358.

[56] E. Olsson, G. Kreiss, S. Zahedi, A conservative level set method for two phase flow II, Journal of Computational Physics 225 (2007) 785-807.

[57] Lord Rayleigh, On the Capillary Phenomena of Jets, Proceedings of the Royal Society of London 29 (1879) $71-97$. 
[58] D. E. Fyfe, E. S. Oran, M. J. Fritts, Surface Tension and Viscosity with Lagrangian Hydrodynamics on a Triangular Mesh, Journal of Computational Physics 76 (1988) 349-384.

[59] D. Igra, K. Takayama, Investigation of Aerodynamic Breakup of a Cylindrical Water Droplet, Atomization and Sprays 11 (2001) 167-185.

[60] D. Igra, M. Sun, Shock-Water Column Interaction, from Initial Impact to Fragmentation Onset, AIAA Journal 48 (2010) 2763-2771.

[61] T. Nonomura, K. Kitamura, K. Fujii, A simple interface sharpening technique with a hyperbolic tangent function applied to compressible two-fluid modeling, Journal of Computational Physics 258 (2014) 95-117.

[62] C.-H. Chang, M.-S. Liou, A robust and accurate approach to computing compressible multiphase flow: Stratified flow model and AUSM+-up scheme, Journal of Computational Physics 225 (2007) 840-873.

[63] J. A. Nicholls, A. A. Ranger, Aerodynamic shattering of liquid drops., AIAA Journal 7 (1969) $285-290$.

[64] J. Han, G. Tryggvason, Secondary breakup of axisymmetric liquid drops. I. Acceleration by a constant body force, Physics of Fluids 11 (1999) 3650.

[65] J. Han, G. Tryggvason, Secondary breakup of a axisymmetric liquid drops. II. Impulsive acceleration, Physics of Fluids 13 (2001) 1554-1565. 